\title{
Small denominators and anomalous behaviour in the incommensurate Hubbard-Holstein model
}

\author{
Vieri Mastropietro* \\ Dipartimento di Matematica, Università di Roma "Tor Vergata" \\ Via della Ricerca Scientifica, I-00133, Roma \\ ABSTRACT. We consider a system of interacting fermions on a chain in \\ a periodic potential incommensurate with the chain spacing. We derive \\ a convergent perturbative expansion, afflicted by a small denominator \\ problem and based on renormalization group, for the two point \\ Schwinger function. We obtain the large distance behavior of the \\ Schwinger function, which is anomalous and described by critical in- \\ dices, related to the gap and the wave function renormalization.
}

\section{Introduction.}

1.1 The Holstein-Hubbard model describes a system of $d=1$ interacting spinless fermions on a chain with unit spacing, moving in a periodic force incommensurate with the spacing of the chain. The hamiltonian is

$$
\begin{gathered}
H=H_{0}+u P+\lambda V \quad H_{0}=\sum_{x, y \in \Lambda} t_{x y} \psi_{x}^{+} \psi_{y}^{-} \\
P=\sum_{x \in \Lambda} \varphi_{x} \psi_{x}^{+} \psi_{x}^{-} \quad V=\sum_{x, y \in \Lambda} v(x-y) \psi_{x}^{+} \psi_{x}^{-} \psi_{y}^{-} \psi_{y}^{+}
\end{gathered}
$$

where $x, y$ are points on the one-dimensional lattice $\Lambda$ with unit spacing, length $L$ and periodic boundary conditions; we shall identify $\Lambda$ with $\{x \in Z:-[L / 2] \leq x \leq[(L-1) / 2]\}$. Moreover the matrix $t_{x y}$ is defined as $t_{x y}=\delta_{x, y}-(1 / 2)\left[\delta_{x, y+1}+\delta_{x, y-1}\right]$, where $\delta_{x, y}$ is the Kronecker delta. The fields $\psi_{x}^{ \pm}$are creation $(+)$and annihilation $(-)$fermionic fields, satisfying periodic boundary conditions: $\psi_{x}^{ \pm}=\psi_{x+L}^{ \pm}$. We set $\mathbf{x}=(x, t),-\beta / 2 \leq t \leq \beta / 2$ for some $\beta>0$; on $t$ antiperiodic boundary conditions are imposed.

The term $P$ represents the interaction of the fermions with a classical field. We are interested in studying potentials which, in the limit $L \rightarrow \infty$, have the form $\varphi_{x}=\bar{\varphi}(2 p x)$, where $\bar{\varphi}$ is a real function on the real line $2 \pi$-periodic and $p / \pi$ is an irrational number, so that the field has a period which is incommensurate with the period of the lattice. We also impose that $\bar{\varphi}(u)$ is of mean zero (its mean value can be absorbed in the chemical potential), even and analytic in $u$, so that

$$
\bar{\varphi}(u)=\sum_{0 \neq n \in Z} \hat{\varphi}_{n} e^{i n u}, \quad\left|\hat{\varphi}_{n}\right| \leq F_{0} e^{-\xi|n|}, \quad \hat{\varphi}_{n}=\hat{\varphi}_{-n}=\hat{\varphi}_{n}^{*} .
$$

At finite volume we need a potential satisfying periodic boundary conditions; hence, at finite $L$, we approximate $\varphi_{x}$ by

$$
\varphi_{x}^{(L)}=\sum_{n=-[L / 2]}^{[(L-1) / 2]} \hat{\varphi}_{n} e^{2 i n p_{L} x},
$$

where $p_{L}$ tends to $p$ as $L \rightarrow \infty$ and is of the form $p_{L}=n_{L} \pi / L$, with $n_{L}$ an integer, relatively prime with respect to $L$. The definition of $p_{L}$ implies that $2 n p_{L}$ is an allowed momentum

* Supported by MURST, Italy. 
(modulo $2 \pi$ ). For technical reasons we need $p_{L}$ verifying the Diophantine property (1.5) uniformly in $L$ and in [BGM1], App. 1, a sequence of numbers verifying (1.5) is constructed. The thermodynamic limit is then taken along a particular diverging sequence of volumes and only along this sequence we can solve the problem (a non infrequent situation in solid state physics, in which some models are solved only with particular boundary conditions).

The term $V$ in the hamiltonian represents the interaction of the fermions by a short range two body potential; in particular we assume $|v(x-y)| \leq C e^{-\kappa|x-y|}$ for suitable values of the constants $C, \kappa$.

Finally if $E_{0}^{n}$ is the ground state energy, i.e. the minimum value of $H$ over the eigenstates $\psi_{n}$ with $n$ particles, the spectral gap is defined as $\Delta=E_{0}^{n+1}+E_{0}^{n-1}-2 E_{0}^{n}$. We denote moreover the infinite volume zero temperature two point Schwinger function by $\lim _{L, \beta \rightarrow \infty} S^{L, \beta}(\mathbf{x} ; \mathbf{y})=S(\mathbf{x} ; \mathbf{y})$, defined in (2.8).

1.2 If $\lambda=0$ the Hamiltonian $H_{0}+u P \equiv \bar{H}$ is quadratic in the Fermi fields and its eigenfunctions are the antisymmetrized product of the one particles wavefunctions $\psi(x)$ of the finite difference Schroedinger equation

$$
-\psi(x+1)-\psi(x-1)+u \varphi_{x} \psi(x)=E \psi(x)
$$

with $\varphi_{x}$ defined as above. It is known that, for $u$ small enough and if $p$ verifies a diophantine condition $\|2 n p\|_{T^{1}} \geq C_{0}|n|^{-\tau}$, for any $0 \neq n \in Z$, there are, for particular values of $E$, eigenfunctions which are quasi Bloch waves of the form $\psi(x)=e^{-i k(E) x} U(k(E), x, u)$, with $U(k(E), x, u)=\bar{U}(k(E), p x, u)$ and $\bar{U} 2 \pi$-periodic in $p x$. In particular this is true if $\| k(E)+$ $n p \|_{T^{1}} \geq C_{0}|n|^{-\tau}, 0 \neq n \in Z$, [DS], or if $k(E)=n p, 0 \neq n \in Z$, [JM],[MP],[E] (strictly speaking these results were proved for the almost equivalent problem of the Schroedinger equation in the continuum with a quasi periodic potential, but one can extend them to this case, see $[\mathrm{BLT}])$. There are, for a generic potential, infinitely many gaps in the spectrum in correspondence of the values of $k(E)=n p$ mod. $2 \pi$, and the spectrum is a Cantor set [E]. These results are obtained by KAM iterative techniques, because the perturbative series for the eigenvectors and eigenvalues of (1.4) are characterized by a small denominator problem quite similar to the one in the series for the invariant tori of classical hamiltonians close to integrable ones. It is useful to compare the above results with their correspondent in the commensurate case, in which $\frac{p}{\pi}$ is a rational number. In this case all the eigenfunctions are Bloch waves, and the gaps are still in correspondence of $k(E)=n p, \bmod .2 \pi$, but the gaps are a finite number, for the rationality of $p / \pi$. In the commensurate case the many body system described by $\bar{H}$ has two physical phases. For values of the chemical potential $\mu$ (hence of the density) outside the gaps of the one particle spectrum, $S(\mathbf{x} ; \mathbf{y})$ decays with a power law for large distances and the (infinite volume) ground state has no gaps $\Delta=0$; this is the metallic phase. Choosing $\mu$ in correspondence of a gap in the one particle spectrum the Schwinger function has a faster than any power decay and the ground state has a gap $\Delta \neq 0$; this is the insulating phase. In the incommensurate case the situation is more complex, but still, see [BGM1], for values of the chemical potential corresponding to a gap in the one particle spectrum $\left(\mu=1-\cos \left(m p_{L}\right), m\right.$ integer $)$ and small $u, S(\mathbf{x} ; \mathbf{y})$ has a faster than any power decay and the ground state has a gap $\Delta \neq 0$. We can call this a quasi-insulating phase.

If we consider also an interaction between fermions we can expect that still there is a quasiinsulating phase, i.e. for suitable values of the chemical potential (hence of the density) $S(\mathbf{x} ; \mathbf{y})$ has a faster than any power decay (but, as we will see, anomalous) and the ground state of $H$ has a gap $\Delta \neq 0$. However there is in general no reason for which the chemical potentials corresponding to the quasi-insulating phase had to be the same as in the $\lambda=$ 0 , and indeed we find that they are different. The right chemical potential to study the quasi-insulating phase is unknown and it is part of the problem; it turns out that $\mu=$ $1-\cos (m p)-\nu$, with $\nu \equiv \nu(\lambda, u)$ is a suitable function such that $\nu(0, u)=0$. Defining the Fermi momentum as the momentum for which the occupation number or some of its 
derivatives are singular, our choice of the chemical potential corresponds to fix the Fermi momentum of the interacting model as $p_{F}=m p, m \in Z^{+}$. One of the main points of our analysis, as well as of the preceding papers starting from [BG], is to use as a physical parameter the Fermi momentum rather than the density. Of course if the formal Luttinger theorem (see for instance [BGL]) holds this is equivalent to fix the density; but its validity is an open problem and there is no need to discuss it here. Fixing the Fermi momentum is very reasonable in a work technically based on renormalization group. Moreover this is the most natural choice from a physical point of view, as discussed in the next section. Nevertheless the problem of fixing the chemical potential to a $\lambda . u$ independent value is an interesting mathematical problem. To obtain results for this problem from ours has to solve an implicit function problem (non trivial as our series are defined, as functions of $p_{F}$, only on $p_{F}$ verifying a diophantine condition), but this will be not addresses here.

1.3 Let we discuss an important physical application of our results, so motivating our choice of fixing the Fermi momentum. Peierls $[\mathrm{P}]$ and Fröhlich $[\mathrm{F}]$ suggested that one-dimensional metals are unstable at low temperature, in the sense that they can lower their energy through a periodic distorsion of the "physical lattice" with period $\frac{\pi}{p_{F}}$, where $p_{F}$ is the Fermi momentum. Such a distorsion is called Charge Density Wave (CDW), as both the "physical lattice" and the electrons charge density form a new periodic structure with period possibly bigger than the original lattice period 1. The CDW is usually represented as a function $\bar{\phi}\left(2 p_{F} x\right)$. Quite interesting is the case of irrational $\frac{p_{F}}{\pi}$, as Fröhlich suggested that, if the period of the CDW is incommensurate with the original period of the lattice, the CDW has an arbitrary phase and so it should carry an electric current. The properties of many compounds are explained in terms of incommensurate CDW, see for instance [L]. In recent times systems with an incommensurate CDW have been reconsidered in the context of high- $T_{c}$ superconductivity; in particular it was suggested that it is crucial to take into account also the interaction between fermions, see $[\mathrm{A}]$. An interacting Fermi system with an incommensurate CDW is then described by the Holstein-Hubbard model (1.1) with $p_{F}=p$, so our results describe this physical situation. We will see that the interaction introduces an anomalous behavior in the model.

There is no mathematical proof that an incommensurate CDW really exists also in presence of an interaction between fermions. One has to show that the ground state energy of (1.1) as a function of $\phi_{x}$ is minimized by $\phi_{x}=\bar{\phi}\left(2 p_{F} x\right)$. Up to now this was proved only if $\lambda=0$ and $p_{F}=\pi / 2$; recent results [BGM2] show that $\bar{\phi}\left(2 p_{F} x\right)$ is a stationary point for the ground state energy of (1.1), if $\lambda=0$ and $p_{F} / \pi$ rational. It is clear, in any event that our results could represent a starting point for this problem when $\lambda \neq 0$, as from them one can write the ground state energy as a well defined expansion (i.e. our work has the analogous role of [BGM1] with respect to [BGM2]).

1.4 Denote by $\|\alpha-\beta\|_{T^{1}}$ the distance on $T^{1}$ of $\alpha, \beta \in T^{1}$, and, for $\mathbf{x}=\left(x, x_{0}\right), \mathbf{y}=$ $\left(y, y_{0}\right) \in R^{2}$, by $|\mathbf{x}-\mathbf{y}|$ the distance $|\mathbf{x}-\mathbf{y}|=\sqrt{(x-y)^{2}+v_{0}\left(x_{0}-y_{0}\right)^{2}}, v_{0}=\sin p_{F}$, $p_{F}=\cos ^{-1}(1-\mu)$. The definition of the two-point Schwinger function $S^{L, \beta}(\mathbf{x} ; \mathbf{y})$ is standard and it will be recalled below, see (2.8). $\mu$ will be the chemical potential of the $\lambda=0$ theory and $\bar{\mu}$ of the $\lambda \neq 0$ theory. Moreover $O(x, y, z)=O(|x|)+O(|y|)+O(|z|)$.

With the above definitions we shall prove the following theorem.

TheOREm Let be $S^{L, \beta}(\mathbf{x} ; \mathbf{y})$ the two point Schwinger function defined in (2.8) with chemical potential $\bar{\mu}$. Let us consider a sequence $L_{i}, i \in Z^{+}$, such that

$$
\lim _{i \rightarrow \infty} L_{i}=\infty, \quad \lim _{i \rightarrow \infty} p_{L_{i}}=p
$$

Let be $\mu=1-\cos \left(\bar{m} p_{L_{i}}\right)$, if $\bar{m}$ is a positive integer such that $\hat{\varphi}_{\bar{m}} \neq 0$, and $p_{L_{i}}$ satisfies the 
diophantine condition

$$
\left\|2 n p_{L_{i}}\right\|_{T^{1}} \geq C_{0}|n|^{-\tau}, \quad 0 \neq n \in Z \quad|n| \leq \frac{L_{i}}{2}
$$

for some positive constants $C_{0}$ and $\tau$ independent of $i$. Then there exists an $\varepsilon_{0}>0$ (independent from $i, \beta)$ and four functions $\nu \equiv \nu(\lambda, u), \eta_{i}(\lambda, u), i=1,2,3$, continuous for $|u|,|\lambda| \leq \varepsilon_{0}$ and $\nu(\lambda, u)=O(\lambda), \eta_{3}(\lambda, u)=\eta_{1}(\lambda, u)\left(1+\eta_{2}(\lambda, u)\right)^{-1}$ and $\eta_{1}(\lambda, u)=\beta_{1} \lambda^{2}+\lambda^{2} O(\lambda, u, \hat{u})$, $\eta_{2}(\lambda, u)=\beta_{2} \lambda+|\lambda| O(\lambda, u, \hat{u})$, with $\beta_{1}, \beta_{2}$ positive generically non vanishing constants, such that, if $\bar{\mu}=\mu-\nu(\lambda, u)$ the following propositions are true.

(i) There exists the limit $\lim _{\substack{\beta \rightarrow \infty \\ i \rightarrow \infty}} S^{L_{i}, \beta}(\mathbf{x} ; \mathbf{y})=S(\mathbf{x} ; \mathbf{y})$

(ii) $S(\mathbf{x} ; \mathbf{y})$ is continuous as a function of $\lambda, u$. Moreover, defining

$$
\hat{u}=\left|u \hat{\varphi}_{\bar{m}}\right|^{1+\eta_{2}} \quad \hat{Z}=\left|u \hat{\varphi}_{\bar{m}}\right|^{-\eta_{1}}
$$

for all $N$ there is $C_{N}$ such that for all $|\mathbf{x}-\mathbf{y}| \geq \hat{u}^{-1}$ ("large distance faster than any power decay")

$$
|S(\mathbf{x}, \mathbf{y})| \leq \frac{1}{\hat{Z}} \hat{u} \frac{C_{N}}{1+(|\hat{u}||\mathbf{x}-\mathbf{y}|)^{N}}
$$

If $1 \leq|\mathbf{x}-\mathbf{y}| \leq \hat{u}^{-1}$ one has ("transient slow decay")

$$
|S(\mathbf{x}, \mathbf{y})| \leq \frac{K_{1}}{|\mathbf{x}-\mathbf{y}|^{1+\eta_{3}}}
$$

with $K_{1}>0$ constant and

$$
S(\mathbf{x}, \mathbf{y})=\frac{1}{|\mathbf{x}-\mathbf{y}|^{\eta_{3}}}\left(g(\mathbf{x}-\mathbf{y})+O(\lambda, u, \hat{u}) C_{2}(\mathbf{x} ; \mathbf{y})\right)
$$

with $g(\mathbf{x}-\mathbf{y})=\lim _{\lambda \rightarrow 0, u \rightarrow 0} S(\mathbf{x} ; \mathbf{y})$ and $\left|C_{2}(\mathbf{x} ; \mathbf{y})\right| \leq \frac{K_{1}}{|\mathbf{x}-\mathbf{y}|}$ for $|\mathbf{x}-\mathbf{y}| \leq \hat{u}^{-1 / 2}$.

(iii)For any $i$ there is a spectral gap $\Delta$ verifying

$$
\Delta \geq \frac{\hat{u}}{2}
$$

1.5 The above theorem shows that also in presence of an interaction between fermions there is a quasi-insulating phase; this is quite remarkable as our results hold also if $\left|u \hat{\varphi}_{\bar{m}}\right|<<|\lambda|$ i.e. if the interaction between fermions is much larger that the amplitude of the incommensurate potential. Like in the non interacting $\lambda=0$ case, one can distinguish two regions in the large distance behavior of the Schwinger function: a transient slow decay and a long distance faster than any power decay. In the first region there is still a power law decay, but with a non universal exponent $1+\eta_{3}$ instead of 1 (anomalous behaviour); in the second region the decay is still faster than any power, like in the $\lambda=0$ case, but the decay rate is $O(\hat{u})$ instead of $O(u)$. We think that the optimal bound in the large distance behaviour is $|S(\mathbf{x}-\mathbf{y})| \leq \frac{c_{1} e^{-c_{2} \hat{u}|\mathbf{x}-\mathbf{y}|}}{|\mathbf{x}-\mathbf{y}|^{1+\eta_{3}}}$, for some constants $c_{i}>0$, and such bound could be possibly proved by a slight improvement of our techniques. The variation of the decay rate suggests that the interacting ground state gap is $O(\hat{u})$, i.e. the ground state has an anomalous gap, and this is confirmed by (1.10), which if the interaction is attractive $(\lambda \leq 0)$ says that the ratio between the bare and interacting gap is $<<1$ and diverging as $u \rightarrow 0$. The phase of the system described by the above theorem can be called anomalous quasi-insulating phase.

The above theorem shows that the system with $\lambda \neq 0$ is not "analitycally close" to the $\lambda=0$ one; the Schwinger functions depends by $\lambda, u$ not analytically and this makes necessary the use of renormalization group methods. The Schwinger functions of the Holstein-Hubbard 
model are studied writing them as a functional Grassmanian integral which is expressed by a perturbative series. The small denominator problem afflicting this series is controlled, like in [BGM1], by using a sort of Bryuno Lemma [B] (see sec. 3.6 below) and using suitable cancellations to face the problem of the resonances. Such cancellations are implemented by renormalization group techniques. The approach we follow is then very closely related to the "direct" methods developed in recent years for proving the KAM theorem by showing the convergence of the Lindstedt series expressing the invariant tori, see [E1],[G1],[CF],[GM]. However in the above quoted papers (including [BGM1]) the perturbative expansion can be expressed in terms of Feynmann graphs which are only tree graphs i.e. with no loops: in fact the KAM theorem is a classical problem and in [BGM1] is discussed the hamiltonian (1.1) with $\lambda=0$ which defines a non interacting model i.e. bilinear in the fields. On the contrary the perturbative series for the Schwinger functions of the Holstein-Hubbard model are expressed in terms of Feynmann graphs with loops. So a small denominator problem in a fully interacting quantum theory is solved, and our work can be considered a quantum KAM theorem. The main idea is to combine such direct methods to study KAM problems with the renormalization group techniques developed to study interacting fermions starting from $[\mathrm{BG}]$ (similar techniques were introduced also in [FMRT]).

1.6 In order to make clearer our results we state an immediate consequence of the theorem proof, see sec. (4.5). We can write

$$
S(\mathbf{x} ; \mathbf{y})=S_{1}(\mathbf{x} ; \mathbf{y})+O(\lambda, u, \hat{u}) S_{2}(\mathbf{x} ; \mathbf{y}),
$$

where, if $\mathbf{k}=\left(k, k_{0}\right)$ :

$$
S_{1}(\mathbf{x} ; \mathbf{y})=g^{(1)}(\mathbf{x} ; \mathbf{y})+\int \frac{d \mathbf{k}}{(2 \pi)^{2}}\left[1-\hat{f}_{1}(\mathbf{k})\right] \phi(k, x, \hat{u}(k)) \phi^{*}(k, y, \hat{u}(k)) \frac{e^{-i k_{0}\left(x_{0}-y_{0}\right)}}{-i k_{0}+\varepsilon(k, \hat{u}(k))}
$$

with, if $p_{F}=\bar{m} p$

$$
\begin{aligned}
g^{(1)}(\mathbf{x} ; \mathbf{y}) & =\int \frac{d \mathbf{k}}{(2 \pi)^{2}} \frac{\hat{f}_{1}(\mathbf{k})}{\hat{Z}(k)} \frac{e^{-i k_{0}\left(x_{0}-y_{0}\right)}}{-i k_{0}+1-\cos k-\mu}, \\
\varepsilon(k, \sigma) & =\left[1-\cos \left(|k|-p_{F}\right)\right] \cos p_{F} \\
& +\operatorname{sign}\left(|k|-p_{F}\right) \sqrt{\left[\sin \left(|k|-p_{F}\right) v_{0}\right]^{2}+\hat{u}(k)^{2}}, \\
\phi(k, x, \hat{u}(k)) & =e^{-i k x} U(k, x, \hat{u}(k)), \\
U(k, x, \hat{u}(k)) & =e^{i \operatorname{sign}(k) p_{F} x}\left[\cos \left(p_{F} x\right) \sqrt{1-\frac{\operatorname{sign}\left(|k|-p_{F}\right) \hat{u}(k)}{\sqrt{\left(\sin \left(|k|-p_{F}\right) v_{0}\right)^{2}+\hat{u}(k)^{2}}}}\right. \\
& \left.-i \operatorname{sign}(k) \sin \left(p_{F} x\right) \sqrt{1+\frac{\operatorname{sign}\left(|k|-p_{F}\right) \hat{u}(k)}{\sqrt{\left(\sin \left(|k|-p_{F}\right) v_{0}\right)^{2}+\hat{u}(k)^{2}}}}\right] .
\end{aligned}
$$

Here $\hat{f}_{1}(\mathbf{k})$ denotes a cutoff function excluding the two points $\mathbf{k}=\left( \pm p_{F}, 0\right)$, (defined in $(2.14))$ and $\hat{u}(k), \hat{Z}(k)$ are two bounded functions such that $|\hat{u}(k)-u|=O(u \lambda)|,| \hat{Z}(k)^{-1}-$ $1 \mid=O(\lambda)$ for ||$k\left|-p_{F}\right|>\frac{p_{F}}{2}$, and $\hat{u}\left(p_{F}\right)=\hat{u}, \hat{Z}\left(p_{F}\right)=\hat{Z}$ given by (1.6); moreover if $\lambda=0$ then $\hat{u}(k)=u, Z(k)=1$ (such functions will be explicitely constructed in sec.(4.5)). Finally $S_{1}(\mathbf{x}, \mathbf{y}), S_{2}(\mathbf{x}, \mathbf{y})$ obeys to the same bound (1.7),(1.8). In the $\lambda=0$ case $\phi(k, x, u)$ is the first order term of a perturbative expansion for the quasi Bloch waves solving (1.4) with $k=p_{F}=\bar{m} p$, see [E], and, as we can expect that $S_{1}$ is the dominant part of $S(\mathbf{x} ; \mathbf{y})$ for large distances, this is in agreement with the results about Schroedinger equation, see sec. (1.2). If $\lambda \neq 0$ again we can write $S(\mathbf{x} ; \mathbf{y})$ as sum of two terms, and $S_{1}(\mathbf{x} ; \mathbf{y})$ is quite similar to the corresponding expression in the $\lambda=0$ case but $\phi(k, x, u)$ is replaced by $\phi(k, x, \hat{u}(k))$ and there is a factor $\frac{1}{\bar{Z}(k)}$ more. It is natural to heuristically interpretate this fact saying that the elementary exitation for small energy in presence of interaction are quasi-particles which are not quasi-Bloch waves but interacting quasi Bloch waves $\frac{1}{\sqrt{\hat{Z}(k)}} \phi(k, x, \hat{u}(k))$. 
1.7 The Holstein-Hubbard model in the commensurate case is discussed (essentially) in [BM2],[BM3], and one can see that the Schwinger function has a similar behaviour. Finally some comments about the spin; the discussion is essentially identical to the one in [BM2], [BM3] for the commensurate case and we do not repeat it. In the spinning case the number of running coupling constants is larger than in the spinless case and the renormalization group flow is more complex. For repulsive interaction $(\lambda>0)$ things do not change while in the opposite case the flow is unbounded and no conclusions can be drawn (except if $\left|\hat{\varphi}_{\bar{m}} u\right|>e^{\frac{-1}{k \lambda}}$, for a suitable positive constant $k$ ); so our results are valid for spinning fermions only if $\lambda>0$ or if $\lambda<0$ but $\left|\hat{\varphi}_{\bar{m}} u\right|>e^{\frac{-1}{k|\lambda|}}$.

\section{Multiscale decomposition and anomalous integration}

2.1 As it is well known, the Schwinger functions can be written as power series in $\lambda$, convergent for $|\lambda| \leq \varepsilon_{\beta}$, for some constant $\varepsilon_{\beta}$ (the only trivial bound of $\varepsilon_{\beta}$ goes to zero, as $\beta \rightarrow \infty$ ). This power expansion is constructed in the usual way in terms of Feynman graphs, by using as free propagator the function

$$
\begin{aligned}
g^{L, \beta}(\mathbf{x} ; \mathbf{y}) & \equiv g^{L, \beta}(\mathbf{x}-\mathbf{y})=\frac{\operatorname{Tr}\left[e^{-\beta\left(H_{0}-\mu N\right)} \mathbf{T}\left(\psi_{\mathbf{x}}^{-} \psi_{\mathbf{y}}^{+}\right)\right]}{\operatorname{Tr}\left[e^{-\beta\left(H_{0}-\mu N\right)}\right]}= \\
& =\frac{1}{L} \sum_{k \in \mathcal{D}_{L}} e^{-i k(x-y)}\left\{\frac{e^{-\tau e(k)}}{1+e^{-\beta e(k)}} \mathbf{1}(\tau>0)-\frac{e^{-(\beta+\tau) e(k)}}{1+e^{-\beta e(k)}} \mathbf{1}(\tau \leq 0)\right\},
\end{aligned}
$$

where $N=\sum_{x \in \Lambda} \psi_{x}^{+} \psi_{x}^{+}, \tau=x_{0}-y_{0}, \mathbf{1}(E)$ denotes the indicator function $(\mathbf{1}(E)=1$, if $E$ is true, $\mathbf{1}(E)=0$ otherwise), $e(k)=1-\cos k-\mu$ and $\mathcal{D}_{L} \equiv\{k=2 \pi n / L, n \in Z,-[L / 2] \leq$ $n \leq[(L-1) / 2]\}$.

It is easy to prove that, if $x_{0} \neq y_{0}$,

$$
g^{L, \beta}(\mathbf{x}-\mathbf{y})=\lim _{M \rightarrow \infty} \frac{1}{L \beta} \sum_{\mathbf{k} \in \mathcal{D}_{L, \beta}} \frac{e^{-i \mathbf{k} \cdot(\mathbf{x}-\mathbf{y})}}{-i k_{0}+\cos p_{F}-\cos k},
$$

where $\mathbf{k}=\left(k, k_{0}\right), \mathbf{k} \cdot \mathbf{x}=k_{0} x_{0}+k x, \mathcal{D}_{L, \beta} \equiv \mathcal{D}_{L} \times \mathcal{D}_{\beta}, \mathcal{D}_{\beta} \equiv\left\{k_{0}=2(n+1 / 2) \pi / \beta, n \in\right.$ $Z,-M \leq n \leq M-1\}$ and $p_{F}$ is the Fermi momentum, defined so that $\cos p_{F}=1-\mu$ and $0 \leq p_{F} \leq \pi$.

Hence, if we introduce a finite set of Grassmanian variables $\left\{\psi_{\mathbf{k}}^{ \pm}\right\}$, one for each of the allowed $\mathbf{k}$ values, and a linear functional $P(d \psi)$ on the generated Grassmanian algebra, such that

$$
\int P(d \psi) \psi_{\mathbf{k}_{1}}^{-} \psi_{\mathbf{k}_{2}}^{+}=L \beta \delta_{\mathbf{k}_{1}, \mathbf{k}_{2}} \hat{g}_{\mathbf{k}_{1}}, \quad \hat{g}_{\mathbf{k}}=\frac{1}{-i k_{0}+\cos p_{F}-\cos k},
$$

we have

$$
\frac{1}{L \beta} \sum_{\mathbf{k} \in \mathcal{D}_{L, \beta}} e^{-i \mathbf{k} \cdot(\mathbf{x}-\mathbf{y})} \hat{g}_{\mathbf{k}}=\int P(d \psi) \psi_{\mathbf{x}}^{-} \psi_{\mathbf{y}}^{+} \equiv g^{L, \beta}(\mathbf{x} ; \mathbf{y}),
$$

where the Grassmanian field $\psi_{\mathbf{x}}$ is defined by

$$
\psi_{\mathbf{x}}^{ \pm}=\frac{1}{L \beta} \sum_{\mathbf{k} \in \mathcal{D}_{L, \beta}} \psi_{\mathbf{k}}^{ \pm} e^{ \pm i \mathbf{k} \cdot \mathbf{x}} .
$$

The "Gaussian measure" $P(d \psi)$ has a simple representation in terms of the "Lebesgue Grassmanian measure" $d \psi d \psi^{+}$, defined as the linear functional on the Grassmanian algebra, such that, given a monomial $Q\left(\psi^{-}, \psi^{+}\right)$in the variables $\psi_{\mathbf{k}}^{-}, \psi_{\mathbf{k}}^{+}$,

$$
\int d \psi^{-} d \psi^{+} Q\left(\psi^{-}, \psi^{+}\right)= \begin{cases}1 & \text { if } Q\left(\psi^{-}, \psi^{+}\right)=\prod_{\mathbf{k}} \psi_{\mathbf{k}}^{-} \psi_{\mathbf{k}}^{+}, \\ 0 & \text { otherwise }\end{cases}
$$


We have

$$
P(d \psi)=\left\{\prod_{\mathbf{k}}\left(L \beta \hat{g}_{\mathbf{k}}\right)\right\} \exp \left\{-\sum_{\mathbf{k}}\left(L \beta \hat{g}_{\mathbf{k}}\right)^{-1} \psi_{\mathbf{k}}^{+} \psi_{\mathbf{k}}^{-}\right\} d \psi^{-} d \psi^{+} .
$$

Note that, since $\left(\psi_{\mathbf{k}}^{-}\right)^{2}=\left(\psi_{\mathbf{k}}^{+}\right)^{2}=0, e^{-z \psi_{\mathbf{k}}^{+} \psi_{\mathbf{k}}}=1-z \psi_{\mathbf{k}}^{+} \psi_{\mathbf{k}}$, for any complex $z$.

By using standard arguments (see, for example, [NO], where a different regularization of the propagator is used), one can show that the Schwinger functions can be calculated as expectations of suitable functions of the Grassmanian field with respect to the "Gaussian measure" $P(d \psi)$. In particular, the two-point Schwinger function can be written, if $x_{0} \neq y_{0}$, as

$$
S^{L, \beta}(\mathbf{x} ; \mathbf{y})=\lim _{M \rightarrow \infty} \frac{\int P(d \psi) e^{-\mathcal{V}(\psi)} \psi_{\mathbf{x}}^{-} \psi_{\mathbf{y}}^{+}}{\int P(d \psi) e^{-\mathcal{V}(\psi)}},
$$

where $\mathcal{V}(\psi)=u P(\psi)+\lambda V(\psi)+\nu N(\psi)$ with

$$
\begin{gathered}
V(\psi)=\sum_{x, y \in \Lambda} \int_{-\beta / 2}^{\beta / 2} d x_{0} \int_{-\beta / 2}^{\beta / 2} d y_{0} v(x-y) \delta\left(x_{0}-y_{0}\right) \psi_{\mathbf{x}}^{+} \psi_{\mathbf{x}}^{-} \psi_{\mathbf{y}}^{-} \psi_{\mathbf{y}}^{+} \\
P(\psi)=\sum_{x \in \Lambda} \int_{-\beta / 2}^{\beta / 2} d x_{0}\left[\varphi_{x}^{(L)} \psi_{\mathbf{x}}^{+} \psi_{\mathbf{x}}^{-}\right] \quad N(\psi)=\sum_{x \in \Lambda} \int_{-\beta / 2}^{\beta / 2} d x_{0} \psi_{\mathbf{x}}^{+} \psi_{\mathbf{x}}^{-} .
\end{gathered}
$$

If $x_{0}=y_{0}, S^{L, \beta}(\mathbf{x} ; \mathbf{y})$ must be defined as the limit of $(2.8)$ as $x_{0}-y_{0} \rightarrow 0^{-}$, as we shall understand always in the following.

2.2 We start by evalutating the partition functioni.e. the denominator of (2.8)

$$
\int P(d \psi) e^{-\mathcal{V}(\psi)}
$$

It is convenient to decompose the Grassmanian integration $P(d \psi)$ into a finite product of independent integrations:

$$
P(d \psi)=\prod_{h=h_{\beta}}^{1} P\left(d \psi^{(h)}\right),
$$

where $h_{\beta}>-\infty$ will be defined below (before (2.19)) This can be done by setting

$$
\psi_{\mathbf{k}}^{ \pm}=\bigoplus_{h=h_{\beta}}^{1} \psi_{\mathbf{k}}^{(h) \pm}, \quad \hat{g}_{\mathbf{k}}=\sum_{h=h_{\beta}}^{1} \hat{g}_{\mathbf{k}}^{(h)}
$$

where $\psi_{\mathbf{k}}^{(h) \pm}$ are families of Grassmanian fields with propagators $\hat{g}_{\mathbf{k}}^{(h)}$ which are defined in the following way.

We introduce a scaling parameter $\gamma>1$ and a function $\chi\left(\mathbf{k}^{\prime}\right) \in C^{\infty}\left(T^{1} \times R\right), \mathbf{k}^{\prime}=\left(k^{\prime}, k_{0}\right)$, such that, if $\left|\mathbf{k}^{\prime}\right| \equiv \sqrt{k_{0}^{2}+\left\|k^{\prime}\right\|_{T^{1}}^{2}}$ :

$$
\chi\left(\mathbf{k}^{\prime}\right)=\chi\left(-\mathbf{k}^{\prime}\right)= \begin{cases}1 & \text { if }\left|\mathbf{k}^{\prime}\right|<t_{0} \equiv a_{0} / \gamma \\ 0 & \text { if }\left|\mathbf{k}^{\prime}\right|>a_{0}\end{cases}
$$

where $a_{0}=\min \left\{p_{F} / 2,\left(\pi-p_{F}\right) / 2\right\}$. This definition is such that the supports of $\chi\left(k-p_{F}, k_{0}\right)$ and $\chi\left(k+p_{F}, k_{0}\right)$ are disjoint and the $C^{\infty}$ function on $T^{1} \times R$

$$
\hat{f}_{1}(\mathbf{k}) \equiv 1-\chi\left(k-p_{F}, k_{0}\right)-\chi\left(k+p_{F}, k_{0}\right)
$$

is equal to 0 , if ||$k \mid-p_{F} \|_{T^{1}}^{2}+k_{0}^{2}<t_{0}^{2}$.

We define also, for any integer $h \leq 0$,

$$
f_{h}\left(\mathbf{k}^{\prime}\right)=\chi\left(\gamma^{-h} \mathbf{k}^{\prime}\right)-\chi\left(\gamma^{-h+1} \mathbf{k}^{\prime}\right) ;
$$


we have, for any $\bar{h}<0$,

$$
\chi\left(\mathbf{k}^{\prime}\right)=\sum_{h=\bar{h}+1}^{0} f_{h}\left(\mathbf{k}^{\prime}\right)+\chi\left(\gamma^{-\bar{h}} \mathbf{k}^{\prime}\right) .
$$

Note that, if $h \leq 0, f_{h}\left(\mathbf{k}^{\prime}\right)=0$ for $\left|\mathbf{k}^{\prime}\right|<t_{0} \gamma^{h-1}$ or $\left|\mathbf{k}^{\prime}\right|>t_{0} \gamma^{h+1}$, and $f_{h}\left(\mathbf{k}^{\prime}\right)=1$, if $\left|\mathbf{k}^{\prime}\right|=t_{0} \gamma^{h}$.

We finally define, for any $h \leq 0$ :

$$
\begin{gathered}
\hat{f}_{h}(\mathbf{k})=f_{h}\left(k-p_{F}, k_{0}\right)+f_{h}\left(k+p_{F}, k_{0}\right), \\
\hat{g}_{\mathbf{k}}^{(h)} \equiv \frac{\hat{f}_{h}(\mathbf{k})}{-i k_{0}+\cos p_{F}-\cos k} .
\end{gathered}
$$

Note that, if $\mathbf{k} \in \mathcal{D}_{L, \beta}$, then $\left|k_{0}\right| \geq \pi / \beta$, implying that $\hat{f}_{h}(\mathbf{k})=0$ for any $h<h_{\beta}=$ $\min \left\{h: t_{0} \gamma^{h+1}>\pi / \beta\right\}$. Hence, if $\mathbf{k} \in \mathcal{D}_{L, \beta}$, the definitions (2.14) and (2.17), together with the identity (2.16), imply that

$$
1=\sum_{h=h_{\beta}}^{1} \hat{f}_{h}(\mathbf{k}) .
$$

The definition (2.17) implies also that, if $h \leq 0$, the support of $\hat{f}_{h}(\mathbf{k})$ is the union of two disjoint sets, $A_{h}^{+}$and $A_{h}^{-}$. In $A_{h}^{+}, k$ is strictly positive and $\left\|k-p_{F}\right\|_{T^{1}} \leq a_{0} \gamma^{h} \leq a_{0}$, while, in $A_{h}^{-}, k$ is strictly negative and $\left\|k+p_{F}\right\|_{T^{1}} \leq a_{0} \gamma^{h}$. Therefore, if $h \leq 0$, we can write $\psi_{\mathbf{k}}^{(h) \pm}$ as the sum of two independent Grassmanian variables $\psi_{\mathbf{k}, \omega}^{(h) \pm}$ with propagator

$$
\int P\left(d \psi^{(h)}\right) \psi_{\mathbf{k}_{1}, \omega_{1}}^{(h)-} \psi_{\mathbf{k}_{2}, \omega_{2}}^{(h)+}=L \beta \delta_{\mathbf{k}_{1}, \mathbf{k}_{2}} \delta_{\omega_{1}, \omega_{2}} \hat{g}_{\omega_{1}}^{(h)}\left(\mathbf{k}_{1}\right),
$$

so that

$$
\begin{gathered}
\psi_{\mathbf{k}}^{(h) \pm}=\bigoplus_{\omega= \pm 1} \psi_{\mathbf{k}, \omega}^{(h) \pm}, \quad \hat{g}_{\mathbf{k}}^{(h)}=\sum_{\omega= \pm 1} \hat{g}_{\omega}^{(h)}(\mathbf{k}) \\
\hat{g}_{\omega}^{(h)}(\mathbf{k})=\frac{\theta(\omega k) \hat{f}_{h}(\mathbf{k})}{-i k_{0}+\cos p_{F}-\cos k}
\end{gathered}
$$

where $\theta(k)$ is the (periodic) step function. If $\omega k>0$, we will write in the following $k=$ $k^{\prime}+\omega p_{F}$, where $k^{\prime}$ is the momentum measured from the Fermi surface and we shall define, if $h \leq 0$,

$$
\tilde{g}_{\omega}^{(h)}\left(\mathbf{k}^{\prime}\right) \equiv \hat{g}_{\omega}^{(h)}(\mathbf{k})=\frac{f_{h}\left(\mathbf{k}^{\prime}\right)}{-i k_{0}+v_{0} \omega \sin k^{\prime}+\left(1-\cos k^{\prime}\right) \cos p_{F}},
$$

where $v_{0}=\sin p_{F}$. We call moreover $\psi_{\mathbf{k}, \omega}^{(\leq k) \pm}=\bigoplus_{h=h_{\beta}}^{k} \psi_{\mathbf{k}, \omega}^{(h) \pm}$ and $g_{\mathbf{k}, \omega}^{(\leq k)}=\sum_{h=h_{\beta}}^{k} \hat{g}_{\mathbf{k}, \omega}^{(h)}$

This kind of decomposition is completely standard in the theory of the $d=1$ Fermi system, starting from $[\mathrm{BG}]$; we repeat it here only for clarity.

We define

$$
e^{-\mathcal{V}^{(0)}\left(\psi^{(\leq 0)}\right)}=\int P\left(d \psi^{(1)}\right) e^{-\mathcal{V}^{(0)}\left(\psi^{(1)}+\psi^{(\leq 0)}\right)}
$$

It is possible to prove, see [BGL], [BGPS] that

$$
\begin{aligned}
& \mathcal{V}^{(0)}\left(\psi^{(\leq 0)}\right)=\lambda \frac{1}{(L \beta)^{4}} \sum_{\mathbf{k}_{1}, \ldots, \mathbf{k}_{4} \in \mathcal{D}_{L, \beta}} \hat{v}\left(\mathbf{k}_{1}-\mathbf{k}_{2}\right) \psi_{\mathbf{k}_{1}}^{(\leq 0)+} \psi_{\mathbf{k}_{2}}^{(\leq 0)-} \psi_{\mathbf{k}_{3}}^{(\leq 0)+} \psi_{\mathbf{k}_{4}}^{(\leq 0)-} \delta\left(\mathbf{k}_{1}+\mathbf{k}_{3}-\mathbf{k}_{2}-\mathbf{k}_{4}\right) \\
& +\frac{1}{L \beta} \sum_{\mathbf{k} \in \mathcal{D}_{L, \beta}}(\nu+F(\mathbf{k})) \psi_{\mathbf{k}}^{(\leq 0)+} \psi_{\mathbf{k}}^{(\leq 0)-}+u \sum_{m=1}^{\infty} \hat{\phi}_{m} \frac{1}{L \beta} \sum_{\mathbf{k} \in \mathcal{D}_{L, \beta}} \psi_{\mathbf{k}}^{(\leq 0)+} \psi_{\mathbf{k}+2 m \mathbf{p}_{L}}^{(\leq 0)-}+\psi_{\mathbf{k}}^{(\leq 0)+} \psi_{\mathbf{k}-2 m \mathbf{p}_{L}}^{(\leq 0)-}
\end{aligned}
$$




$$
+\sum_{n=1}^{\infty} \sum_{m=1}^{\infty} \frac{1}{(L \beta)^{n}} \sum_{\mathbf{k}_{1}, \ldots, \mathbf{k}_{n} \in \mathcal{D}_{L, \beta}} \psi_{\mathbf{k}_{1}}^{(\leq 0) \sigma_{1}} \ldots \psi_{\mathbf{k}_{n}}^{(\leq 0) \sigma_{n}} W_{n, m}^{0}\left(\mathbf{k}_{1}, \ldots, \mathbf{k}_{n}\right) \delta\left(\sum_{i=1}^{n} \sigma_{i} \mathbf{k}_{i}+2 m \mathbf{p}_{L}\right)
$$

where $\sigma_{i}= \pm,|F(\mathbf{k})| \leq C|\lambda|$ and the kernels $W_{n, m}^{0}\left(\mathbf{k}_{1}, \ldots, \mathbf{k}_{n} ; z\right)$ are $C^{\infty}$ bounded functions such that $W_{n, m}^{0}=W_{n,-m}^{0}$ and $\left|W_{n, m}^{0}\right| \leq C^{n} z^{\max (2, n / 2-1)}$ if $z=\operatorname{Max}(|\lambda|, u,|\nu|)$; moreover $\delta(\mathbf{k})=L \beta \delta_{k_{0}} \delta_{k}$ and $\mathbf{p}_{L}=\left(p_{L}, 0\right)$.

2.3 The integration is performed iteratively, setting $Z_{0}=1$, in the following way: once that the fields $\psi^{0}, \ldots, \psi^{h+1}$ have been integrated we have

$$
\int P_{Z_{h}}\left(d \psi^{(\leq h)}\right) e^{-\mathcal{V}^{(h)}\left(\sqrt{Z_{h}} \psi^{(\leq h)}\right)}
$$

with, if $\mathbf{p}_{F}=\left(p_{F}, 0\right)$ and $C_{h}\left(\mathbf{k}^{\prime}\right)^{-1}=\sum_{j=h_{\beta}}^{h} f_{j}\left(\mathbf{k}^{\prime}\right)$ and, up to a constant:

$$
\begin{aligned}
P_{Z_{h}}\left(d \psi^{(\leq h)}\right)= & \prod_{\mathbf{k}} \prod_{\omega= \pm 1} d \psi_{\mathbf{k}^{\prime}+\omega \mathbf{p}_{F}, \omega}^{(\leq h)+} d \psi_{\mathbf{k}^{\prime}+\omega \mathbf{p}_{F}, \omega}^{(\leq h)-} \\
\exp \{ & -\sum_{\omega= \pm 1} \frac{1}{L \beta} \sum_{\mathbf{k}^{\prime} \in \mathcal{D}_{L, \beta}} C_{h}\left(\mathbf{k}^{\prime}\right) Z_{h}\left[\left(-i k_{0}-\left(\cos k^{\prime}-1\right) \cos p_{F}+\omega v_{0} \sin k^{\prime}\right)\right. \\
& \left.\left.\psi_{\mathbf{k}^{\prime}+\omega \mathbf{p}_{F}, \omega}^{(\leq 0)+} \psi_{\mathbf{k}^{\prime}+\omega \mathbf{p}_{F}, \omega}^{(\leq 0)-}+\sigma_{h}\left(\mathbf{k}^{\prime}\right) \psi_{\mathbf{k}^{\prime}+\omega \mathbf{p}_{F}, \omega}^{(\leq 0)+} \psi_{\mathbf{k}^{\prime}-\omega \mathbf{p}_{F},-\omega}^{(\leq 0)-}\right]\right\}
\end{aligned}
$$

It is convenient, for reasons which will be clear below, to split $\mathcal{V}^{(h)}$ as $\mathcal{L} \mathcal{V}^{(h)}+\mathcal{R} \mathcal{V}^{(h)}$, with $\mathcal{R}=1-\mathcal{L}$ and $\mathcal{L}$, the localization operator, is a linear operator defined in the following way:

1) If $n>4$ then

$\mathcal{L}\left\{\frac{1}{(L \beta)^{n}} \sum_{\mathbf{k}_{1}^{\prime}, \ldots, \mathbf{k}_{n}^{\prime} \in \mathcal{D}_{L, \beta}} W_{n, m}^{h}\left(\mathbf{k}_{1}^{\prime}+\omega_{1} \mathbf{p}_{F}, \ldots\right) \prod_{i=1}^{n} \psi_{\mathbf{k}_{i}^{\prime}+\omega_{i} \mathbf{p}_{F}, \omega_{i}}^{(\leq h) \sigma_{i}} \delta\left(\sum_{i=1}^{n} \sigma_{i}\left(\mathbf{k}_{i}^{\prime}+\omega_{i} \mathbf{p}_{F}\right)+2 m \mathbf{p}_{L}\right)\right\}=0$

2) If $n=4$ then

$$
\begin{aligned}
& \mathcal{L}\left\{\frac{1}{(L \beta)^{4}} \sum_{\mathbf{k}_{1}^{\prime}, \ldots, \mathbf{k}_{4}^{\prime} \in \mathcal{D}_{L, \beta}} W_{4, m}^{h}\left(\mathbf{k}_{1}^{\prime}+\omega_{1} \mathbf{p}_{F}, \ldots\right)\left[\prod_{i=1}^{4} \psi_{\mathbf{k}_{i}^{\prime}+\omega_{i} \mathbf{p}_{F}, \omega_{i}}^{(\leq h) \sigma_{i}}\right] \delta\left(\sum_{i=1}^{4} \sigma_{i}\left(\mathbf{k}_{i}^{\prime}+\omega_{i} \mathbf{p}_{F}\right)+2 m \mathbf{p}_{L}\right)\right\} \\
& =\delta_{\sum_{i=1}^{4} \sigma_{i} \omega_{i} p_{F}+2 m p_{L}, 0} \frac{1}{(L \beta)^{4}} \sum_{\mathbf{k}_{1}^{\prime}, \ldots, \mathbf{k}_{4}^{\prime} \in \mathcal{D}_{L, \beta}} W_{4, m}^{h}\left(\omega_{1} \mathbf{p}_{F}, \ldots, \omega_{4} \mathbf{p}_{F}\right)\left[\prod_{i=1}^{4} \psi_{\mathbf{k}_{i}^{\prime}+\omega_{i} \mathbf{p}_{F}, \omega_{i}}^{(\leq h) \sigma_{i}}\right] \delta\left(\sum_{i=1}^{4} \sigma_{i} \mathbf{k}_{i}^{\prime}\right)
\end{aligned}
$$

3)If $n=2$ then

$$
\begin{gathered}
\mathcal{L}\left\{\frac{1}{(L \beta)^{2}} \sum_{\mathbf{k}_{1}^{\prime}, \mathbf{k}_{2}^{\prime} \in \mathcal{D}_{L, \beta}} W_{2, m}^{h}\left(\mathbf{k}_{1}^{\prime}+\omega_{1} \mathbf{p}_{F}, \mathbf{k}_{2}^{\prime}+\omega_{2} \mathbf{p}_{F}\right)\left[\prod_{i=1}^{2} \psi_{\mathbf{k}_{i}^{\prime}+\omega_{i} \mathbf{p}_{F}, \omega_{i}}^{(\leq 0) \sigma_{i}}\right]\right. \\
\left.\delta\left(\sum_{i=1}^{2} \sigma_{i}\left(\mathbf{k}_{i}^{\prime}+\omega_{i} \mathbf{p}_{F}\right)+2 m \mathbf{p}_{L}\right)\right\}=\delta_{\left(\omega_{1}-\omega_{2}\right) p_{F}+2 m p_{L}, 0} \frac{1}{(L \beta)} \sum_{\mathbf{k}^{\prime} \in \mathcal{D}_{L, \beta}}\left[W_{2, m}^{h}\left(\omega_{1} \mathbf{p}_{F}, \omega_{2} \mathbf{p}_{F}\right)+\right. \\
\left.+\omega_{1} E\left(k^{\prime}+\omega_{1} p_{F}\right) \partial_{k} W_{2, m}^{h}\left(\omega_{1} \mathbf{p}_{F}, \omega_{2} \mathbf{p}_{F}\right)+k^{0} \partial_{k_{0}} W_{2, m}^{h}\left(\omega_{1} \mathbf{p}_{F}, \omega_{2} \mathbf{p}_{F}\right)\right]\left[\prod_{i=1}^{2} \psi_{\mathbf{k}_{i}^{\prime}+\omega_{i} \mathbf{p}_{F}, \omega_{i}}^{(\leq h) \sigma_{i}}\right]
\end{gathered}
$$


where $E\left(k^{\prime}+\omega p_{F}\right)=v_{0} \omega \sin k^{\prime}+\left(1-\cos k^{\prime}\right) \cos p_{F}$ and the symbol $\partial_{k}, \partial_{k_{0}}$ means discrete derivatives. Note that the r.h.s of $(2.26),(2.27)$ are vanishing unless $\sum_{i} \sigma_{i} \omega_{i} p_{F}+2 m p_{L}=0$ $\bmod .2 \pi$.

We can write then $\mathcal{L} \mathcal{V}^{(h)}$ in the following way:

$$
\mathcal{L V}^{(h)}(\psi)=\gamma^{h} n_{h} F_{\nu}^{(\leq h)}+s_{h} F_{\sigma}^{(\leq h)}+z_{h} F_{\zeta}^{(\leq h)}+a_{h} F_{\alpha}^{(\leq h)}+i_{h} F_{\iota}^{(\leq h)}+t_{h} F_{\tau}^{(\leq h)}+l_{h} F_{\lambda}^{(\leq h)}
$$

where

$$
\begin{aligned}
& F_{\nu}^{(\leq h)}=\sum_{\omega= \pm 1} \frac{1}{(L \beta)} \sum_{\mathbf{k}^{\prime} \in \mathcal{D}_{L, \beta}} \psi_{\mathbf{k}^{\prime}+\omega \mathbf{p}_{F}, \omega}^{(\leq h)+} \psi_{\mathbf{k}^{\prime}+\omega \mathbf{p}_{F}, \omega}^{(\leq h)-} \\
& F_{\sigma}^{(\leq h)}=\sum_{\omega= \pm 1} \frac{1}{(L \beta)} \sum_{\mathbf{k}^{\prime} \in \mathcal{D}_{L, \beta}} \psi_{\mathbf{k}^{\prime}+\omega \mathbf{p}_{F}, \omega}^{(\leq h)+} \psi_{\mathbf{k}^{\prime}-\omega \mathbf{p}_{F},-\omega}^{(\leq h)-} \\
& F_{\alpha}^{(\leq h)}=\sum_{\omega= \pm 1} \frac{1}{(L \beta)} \sum_{\mathbf{k}^{\prime} \in \mathcal{D}_{L, \beta}} E\left(k^{\prime}+\omega p_{F}\right) \psi_{\mathbf{k}^{\prime}+\omega \mathbf{p}_{F}, \omega}^{(\leq h)+} \psi_{\mathbf{k}^{\prime}+\omega \mathbf{p}_{F}, \omega}^{(\leq h)-} \\
& F_{\zeta}^{(\leq h)}=\sum_{\omega= \pm 1} \frac{1}{(L \beta)} \sum_{\mathbf{k}^{\prime} \in \mathcal{D}_{L, \beta}}\left(-i k_{0}\right) \psi_{\mathbf{k}^{\prime}+\omega \mathbf{p}_{F}, \omega}^{(\leq h)+} \psi_{\mathbf{k}^{\prime}+\omega \mathbf{p}_{F}, \omega}^{(\leq h)-} \\
& F_{\iota}^{(\leq h)}=\sum_{\omega= \pm 1} \frac{1}{(L \beta)} \sum_{\mathbf{k}^{\prime} \in \mathcal{D}_{L, \beta}} E\left(k^{\prime}+\omega p_{F}\right) \psi_{\mathbf{k}^{\prime}+\omega \mathbf{p}_{F}, \omega}^{(\leq h)+} \psi_{\mathbf{k}^{\prime}-\omega \mathbf{p}_{F},-\omega}^{(\leq h)-} \\
& F_{\tau}^{(\leq h)}=\sum_{\omega= \pm 1} \frac{1}{(L \beta)} \sum_{\mathbf{k}^{\prime} \in \mathcal{D}_{L, \beta}}\left(-i k_{0}\right) \psi_{\mathbf{k}^{\prime}+\omega \mathbf{p}_{F}, \omega}^{(\leq h)+} \psi_{\mathbf{k}^{\prime}-\omega \mathbf{p}_{F},-\omega}^{(\leq h)-} \\
& F_{\lambda}^{(\leq h)}=\frac{1}{(L \beta)^{4}} \sum_{\mathbf{k}_{1}^{\prime}, \ldots, \mathbf{k}_{4}^{\prime} \in \mathcal{D}_{L, \beta}} \psi_{\mathbf{k}_{1}^{\prime}+\mathbf{p}_{F}, 1}^{(\leq h)+} \psi_{\mathbf{k}_{1}^{\prime}-\mathbf{p}_{F},-1}^{(\leq h)+} \psi_{\mathbf{k}_{3}^{\prime}+\mathbf{p}_{F}, 1}^{(\leq 0)-} \psi_{\mathbf{k}_{4}^{\prime}-\mathbf{p}_{F},-1}^{(\leq 0)-} \delta\left(\sum_{i=1}^{4} \sigma_{i} \mathbf{k}_{i}\right)
\end{aligned}
$$

and $\lambda_{0}=\lambda\left(\hat{v}(0)-\hat{v}\left(2 p_{F}\right)\right)+O\left(\lambda^{2}\right), s_{0}=u \hat{\phi}_{\bar{m}}+O\left(u \lambda^{2}\right), t_{0}, i_{0}=O\left(u \lambda^{2}\right), a_{0}, z_{0}=O\left(\lambda^{2}\right)$, $n_{0}=\nu+O\left(\lambda^{2}\right)$. This follows from the antisimmetry properties of the Grassman variables and the Kroneker deltas in the r.h.s. of (2.26),(2.27).

We write, if $\mathcal{N}_{h}$ is a constant

$$
\int P_{Z_{h}}\left(d \psi^{(\leq h)}\right) e^{-\mathcal{V}^{(h)}\left(\sqrt{Z_{h}} \psi^{(\leq h)}\right)}=\frac{1}{\mathcal{N}_{h}} \int \tilde{P}_{Z_{h-1}}\left(d \psi^{(\leq h)}\right) e^{-\tilde{\mathcal{V}}^{(h)}\left(\sqrt{Z_{h}} \psi^{(\leq h)}\right)}
$$

where

$$
\begin{aligned}
& \tilde{P}_{Z_{h-1}}\left(d \psi^{(\leq h)}\right)=\prod_{\mathbf{k}} \prod_{\omega= \pm 1} d \psi_{\mathbf{k}^{\prime}+\omega \mathbf{p}_{F}, \omega}^{(\leq h)+} d \psi_{\mathbf{k}^{\prime}+\omega \mathbf{p}_{F}, \omega}^{(\leq h)-} \\
& \exp \left\{-\sum_{\omega= \pm 1} \frac{1}{L \beta} \sum_{\mathbf{k}^{\prime} \in \mathcal{D}_{L, \beta}} C_{h}\left(\mathbf{k}^{\prime}\right) Z_{h-1}\left(\mathbf{k}^{\prime}\right)\left[\left(-i k_{0}-\left(\cos k^{\prime}-1\right) \cos p_{F}+\omega v_{0} \sin k^{\prime}\right)\right.\right. \\
& \left.\left.\psi_{\mathbf{k}^{\prime}+\omega \mathbf{p}_{F}, \omega}^{(\leq 0)+} \psi_{\mathbf{k}^{\prime}+\omega \mathbf{p}_{F}, \omega}^{(\leq 0)-}+\sigma_{h-1}\left(\mathbf{k}^{\prime}\right) \psi_{\mathbf{k}^{\prime}+\omega \mathbf{p}_{F}, \omega}^{(\leq 0)+} \psi_{\mathbf{k}^{\prime}-\omega \mathbf{p}_{F},-\omega}^{(\leq 0)-}\right]\right\},
\end{aligned}
$$

with $Z_{h-1}\left(\mathbf{k}^{\prime}\right)=Z_{h}\left(1+C_{h}^{-1}\left(\mathbf{k}^{\prime}\right) z_{h}\right), Z_{h-1}\left(\mathbf{k}^{\prime}\right) \sigma_{h-1}\left(\mathbf{k}^{\prime}\right)=Z_{h}\left(\sigma_{h}\left(\mathbf{k}^{\prime}\right)+C_{h}^{-1}\left(\mathbf{k}^{\prime}\right) s_{h}\right)$ and $\tilde{\mathcal{V}}^{(h)}=\mathcal{L} \tilde{\mathcal{V}}^{(h)}+(1-\mathcal{L}) \mathcal{V}^{(h)}$ with

$$
\mathcal{L} \tilde{\mathcal{V}}^{(h)}(\psi)=\gamma^{h} n_{h} F_{\nu}^{(\leq h)}+\left(a_{h}-z_{h}\right) F_{\alpha}^{(\leq h)}+i_{h} F_{\iota}^{(\leq h)}+t_{h} F_{\tau}^{(\leq h)}+l_{h} F_{\lambda}^{(\leq h)}
$$


The r.h.s of (2.29) can be written as

$$
\frac{1}{\mathcal{N}_{h}} \int P_{Z_{h-1}}\left(d \psi^{(\leq h-1)}\right) \int \tilde{P}_{Z_{h-1}}\left(d \psi^{(h)}\right) e^{-\tilde{\mathcal{V}}^{(h)}\left(\sqrt{Z_{h}} \psi^{(\leq h)}\right)},
$$

where $P_{Z_{h-1}}\left(d \psi^{(\leq h-1)}\right)$ and $\tilde{P}_{Z_{h-1}}\left(d \psi^{(h)}\right)$ are given by $(2.30)$ with $Z_{h-1}\left(\mathbf{k}^{\prime}\right)$ replaced by $Z_{h-1}(0) \equiv Z_{h-1}$ and $C_{h}\left(\mathbf{k}^{\prime}\right)$ replaced with $C_{h-1}\left(\mathbf{k}^{\prime}\right)$ and $\tilde{f}_{h}^{-1}\left(\mathbf{k}^{\prime}\right)$ respectively, if

$$
\tilde{f}_{h}\left(\mathbf{k}^{\prime}\right)=Z_{h-1}\left[\frac{C_{h}^{-1}\left(\mathbf{k}^{\prime}\right)}{Z_{h-1}\left(\mathbf{k}^{\prime}\right)}-\frac{C_{h-1}^{-1}\left(\mathbf{k}^{\prime}\right)}{Z_{h-1}}\right]
$$

and $\psi^{(\leq h)}$ replaced with $\psi^{(\leq h-1)}$ and $\psi^{(h)}$ respectively. Note that $\tilde{f}_{h}\left(\mathbf{k}^{\prime}\right)$ is a compact support function, with support of width $O\left(\gamma^{h}\right)$ and far $O\left(\gamma^{h}\right)$ from the "singularity" i.e. $\mathbf{p}_{F}$.

The Grassmanian integration $\tilde{P}_{Z_{h-1}}\left(d \psi^{(h)}\right)$ has propagator

$$
\frac{g^{(h)}(\mathbf{x} ; \mathbf{y})}{Z_{h-1}}=\sum_{\omega, \omega^{\prime}= \pm 1} e^{-i\left(\omega x-\omega^{\prime} y\right) p_{F}} \frac{g_{\omega, \omega^{\prime}}^{(h)}(\mathbf{x} ; \mathbf{y})}{Z_{h-1}}
$$

if

$$
\frac{g_{\omega, \omega^{\prime}}^{(h)}(\mathbf{x} ; \mathbf{y})}{Z_{h-1}}=\int \tilde{P}_{Z_{h-1}}\left(d \psi^{(h)}\right) \psi_{\mathbf{x}, \omega}^{(h)-} \psi_{\mathbf{y}, \omega^{\prime}}^{(h)+}
$$

is given by

$$
g_{\omega, \omega^{\prime}}^{(h)}(\mathbf{x} ; \mathbf{y})=\frac{1}{L \beta} \sum_{\mathbf{k}^{\prime} \in \mathcal{D}_{L, \beta}} e^{-i \mathbf{k}^{\prime} \cdot(\mathbf{x}-\mathbf{y})} \tilde{f}_{h}\left(\mathbf{k}^{\prime}\right)\left[T_{h}^{-1}\left(\mathbf{k}^{\prime}\right)\right]_{\omega, \omega^{\prime}},
$$

where the $2 \times 2$ matrix $T_{h}\left(\mathbf{k}^{\prime}\right)$ has elements

$$
\left\{\begin{array}{l}
{\left[T_{h}\left(\mathbf{k}^{\prime}\right)\right]_{1,1}=\left(-i k_{0}-\left(\cos k^{\prime}-1\right) \cos p_{F}+v_{0} \sin k^{\prime}\right)} \\
{\left[T_{h}\left(\mathbf{k}^{\prime}\right)\right]_{1,2}=\left[T_{h}\left(\mathbf{k}^{\prime}\right)\right]_{2,1}=\sigma_{h-1}\left(\mathbf{k}^{\prime}\right)} \\
{\left[T_{h}\left(\mathbf{k}^{\prime}\right)\right]_{2,2}=\left(-i k_{0}-\left(\cos k^{\prime}-1\right) \cos p_{F}-v_{0} \sin k^{\prime}\right)}
\end{array}\right.
$$

which is well defined on the support of $\tilde{f}_{h}\left(\mathbf{k}^{\prime}\right)$, so that, if we set

$$
A_{h}\left(\mathbf{k}^{\prime}\right)=\operatorname{det} T_{h}\left(\mathbf{k}^{\prime}\right)=\left[-i k_{0}-\left(\cos k^{\prime}-1\right) \cos p_{F}\right]^{2}-\left(v_{0} \sin k^{\prime}\right)^{2}-\left[\sigma_{h-1}\left(\mathbf{k}^{\prime}\right)\right]^{2},
$$

then

$$
T_{h}^{-1}\left(\mathbf{k}^{\prime}\right)=\frac{1}{A_{h}\left(\mathbf{k}^{\prime}\right)}\left(\begin{array}{ll}
{\left[\tau_{h}\left(\mathbf{k}^{\prime}\right)\right]_{1,1}} & {\left[\tau_{h}\left(\mathbf{k}^{\prime}\right)\right]_{1,2}} \\
{\left[\tau_{h}\left(\mathbf{k}^{\prime}\right)\right]_{2,1}} & {\left[\tau_{h}\left(\mathbf{k}^{\prime}\right)\right]_{2,2}}
\end{array}\right)
$$

with

$$
\left\{\begin{array}{l}
{\left[\tau_{h}\left(\mathbf{k}^{\prime}\right)\right]_{1,1}=\left[-i k_{0}-\left(\cos k^{\prime}-1\right) \cos p_{F}-v_{0} \sin k^{\prime}\right],} \\
{\left[\tau_{h}\left(\mathbf{k}^{\prime}\right)\right]_{1,2}=\left[\tau_{h}\left(\mathbf{k}^{\prime}\right)\right]_{2,1}=-\sigma_{h-1}\left(\mathbf{k}^{\prime}\right),} \\
{\left[\tau_{h}\left(\mathbf{k}^{\prime}\right)\right]_{2,2}=\left[-i k_{0}-\left(\cos k^{\prime}-1\right) \cos p_{F}+v_{0} \sin k^{\prime}\right] .}
\end{array}\right.
$$

Note that $\sigma_{h}\left(\mathbf{k}^{\prime}\right)$ is a smooth function on $T^{1} \times R$ and, if $\mathbf{k}^{\prime}$ varies in the support of $C_{h}^{-1}\left(\mathbf{k}^{\prime}\right)$ then there exists two positive constants $c_{1}, c_{2}$ such that:

$$
c_{1} \sigma_{h} \leq \sigma_{h}\left(\mathbf{k}^{\prime}\right) \leq c_{2} \sigma_{h}
$$

if $\sigma_{h} \equiv \sigma_{h}(0)$, as $C_{k}^{-1}\left(\mathbf{k}^{\prime}\right)=1$ for $k \geq h+1$.

The large distance behaviour of the propagator (2.33) is given by the following lemma, see [BM2]:

2.4LEMMA The propagator $g_{\omega, \omega^{\prime}}^{(h)}(\mathbf{x} ; \mathbf{y})$ can be written as:

$$
g_{\omega, \omega}^{(h)}(\mathbf{x} ; \mathbf{y})=g_{L ; \omega}^{(h)}(\mathbf{x} ; \mathbf{y})+C_{1}^{(h)}(\mathbf{x} ; \mathbf{y})+C_{2}^{(h)}(\mathbf{x} ; \mathbf{y})
$$


with

$$
g_{L ; \omega}^{(h)}(\mathbf{x} ; \mathbf{y})=\int d \mathbf{k} \frac{e^{-i \mathbf{k}(\mathbf{x}-\mathbf{y})}}{-i k_{0}+\omega v_{0} k^{\prime}} \tilde{f}_{h}\left(\mathbf{k}^{\prime}\right)
$$

For any integer $N>1$ and for $|x-y| \leq \frac{L}{2},\left|x_{0}-y_{0}\right| \leq \frac{\beta}{2}$ it holds $\left|C_{1}^{(h)}(\mathbf{x} ; \mathbf{y})\right| \leq$ $\frac{\gamma^{h}\left(\max \left\{\gamma^{h}, L^{-1}\right\}\right) C_{N}}{1+\left(\gamma^{h}(\mathbf{x}-\mathbf{y})\right)^{N}}$ and $\left|C_{2}^{(h)}(\mathbf{x} ; \mathbf{y})\right| \leq\left|\frac{\sigma^{h}}{\gamma^{h}}\right|^{2} \frac{\left(\max \left\{\gamma^{h}, L^{-1}\right\}\right) C_{N}}{1+\left(\gamma^{h}(\mathbf{x}-\mathbf{y})\right)^{N}}$.

Moreover

$$
\left|g_{\omega,-\omega}^{(h)}(\mathbf{x} ; \mathbf{y})\right| \leq\left|\frac{\sigma^{h}}{\gamma^{h}}\right| \frac{\left(\max \left\{\gamma^{h}, L^{-1}\right\}\right) C_{N}}{1+\left(\gamma^{h}(\mathbf{x}-\mathbf{y})\right)^{N}}
$$

Note that $g_{L ; \omega}^{(h)}(\mathbf{x} ; \mathbf{y})$ coincides with the propagator "at scale $\gamma^{h}$ " of the Luttinger model, see $[\mathrm{BeGM}]$. This remark will be crucial for studying the Renormalization group flow, see sec.4. For the proof of the bounds, one can proceed as in the proof of lemma 2.8 below.

2.5 We rescale the fields so that

$$
\frac{1}{\mathcal{N}_{h}} \int P_{Z_{h-1}}\left(d \psi^{(\leq h-1)}\right) \int \tilde{P}_{Z_{h-1}}\left(d \psi^{(h)}\right) e^{-\hat{\mathcal{V}}^{(h)}\left(\sqrt{Z_{h-1}} \psi^{(\leq h)}\right)}
$$

so that

$$
\mathcal{L} \hat{\mathcal{V}}^{(h)}(\psi)=\gamma^{h} \nu_{h} F_{\nu}^{(\leq h)}+\delta_{h} F_{\alpha}^{(\leq h)}+\iota_{h} F_{\iota}^{(\leq h)}+\tau_{h} F_{\tau}^{(\leq h)}+\lambda_{h} F_{\lambda}^{(\leq h)} .
$$

where by definition

$$
\nu_{h}=\frac{Z_{h}}{Z_{h-1}} n_{h} ; \quad \delta_{h}=\frac{Z_{h}}{Z_{h-1}}\left(a_{h}-z_{h}\right) ; \quad \tau_{h}=\frac{Z_{h}}{Z_{h-1}} t_{h} ; \quad \iota_{h}=\frac{Z_{h}}{Z_{h-1}} i_{0} ; \quad \lambda_{h}=\left(\frac{Z_{h}}{Z_{h-1}}\right)^{2} l_{h}
$$

We call the set $\vec{v}_{h}=\left(\nu_{h}, \delta_{h}, \tau_{h}, \iota_{h}, \lambda_{h}\right)$ running coupling constants.

We perform the integration

$$
\int \tilde{P}_{Z_{h-1}}\left(d \psi^{(h)}\right) e^{-\hat{\mathcal{V}}^{(h)}\left(\sqrt{Z_{h-1}} \psi^{(\leq h)}\right)}=e^{-\mathcal{V}^{h-1}\left(\sqrt{Z_{h-1}} \psi^{(\leq h-1)}\right)+\tilde{E}_{h}},
$$

where $\tilde{E}_{h}$ is a suitable constant and

$$
\begin{gathered}
\mathcal{L} \mathcal{V}^{(h-1)}(\psi)=\gamma^{h-1} n_{h-1} F_{\nu}^{\leq(h-1)}+s_{h-1} F_{\sigma}^{\leq(h-1)}+a_{h-1} F_{\alpha}^{(\leq h-1)}+ \\
z_{h-1} F_{\zeta}^{(\leq h-1)}+i_{h-1} F_{\iota}^{(\leq h-1)}+t_{h-1} F_{\tau}^{(\leq h-1)}+l_{h-1} F_{\lambda}^{(\leq-1)},
\end{gathered}
$$

Note that the above procedure allows us to write the running coupling constants $\vec{v}_{h}$ in terms of $\vec{v}_{k}, k \geq h+1$

$$
\vec{v}_{h}=\vec{\beta}\left(\vec{v}_{h+1}, \ldots, \vec{v}_{0}\right)
$$

The function $\vec{\beta}\left(\vec{v}_{h+1}, \ldots, \vec{v}_{0}\right)$ is called Beta function.

The effective potential $\mathcal{V}^{(h)}(\psi)$ is a sum of terms of the form

$$
\frac{1}{(L \beta)^{n}} \sum_{\mathbf{k}_{1}^{\prime}, \ldots, \mathbf{k}_{n}^{\prime} \in \mathcal{D}_{L, \beta}} \delta\left(\sum_{i=1}^{n} \sigma_{i}\left(\mathbf{k}_{i}^{\prime}+\omega_{i} \mathbf{p}_{F}\right)+2 m \mathbf{p}_{L}\right) W_{n, m}^{h}\left(\mathbf{k}_{1}^{\prime}, . ., \mathbf{k}_{n}^{\prime} ;_{n}^{\prime} ;\{\omega\}\right) \prod_{i=1}^{n} \psi_{\mathbf{k}_{i}^{\prime}+\omega_{i} \mathbf{p}_{F}, \omega_{i}}^{\sigma_{i}(\leq h)}
$$

2.6 Let we explain the main motivations of the integration procedure discussed above. In a renormalization group approach one has to identify the relevant, marginal and irrelevant interactions. By a power counting argument one sees that the terms bilinear in the fields are relevant and the quartic terms (or the bilinear ones with a derivative respect to some coordinate acting on the fields) are marginal. However there are $O(L)$ many different terms 
bilinear or quartic in the fields, depending on the value of $m$ in (2.46), and so it seems that there are $O(L)$ different running coupling constants, and their renormalization group flow seems impossible to study. However it will turn out that only a small subset of bilinear or quartic interactions are really relevant or marginal: the terms such that $\sum_{i} \sigma_{i} \boldsymbol{\omega}_{i} p_{F}+2 m p_{L}=$ 0 mod. $2 \pi$. Then we define the $\mathcal{L}$ operator $(2.26),(2.27)$ just to extract such terms from the effective potential. The reason why the terms such that $\sum_{i} \sigma_{i} \boldsymbol{\omega}_{i} p_{F}+2 m p_{L} \neq 0$ are irrelevant is quite clear in the commensurate case i.e. if $p / \pi$ is a rational number. In this case in fact $\left\|\sum_{i=1}^{n} \sigma_{i} \boldsymbol{\omega}_{i} p_{F}+2 m p_{L}\right\|_{T^{1}}$ is greater than some positive number, and this, by the momentum conservation, means that the momenta of the fields cannot be all at the same time closer than some fixed quantity to the singularity of the propagator. In the incommensurate case however due to irrationality of $p / \pi$ the factor $\left\|\sum_{i=1}^{n} \sigma_{i} \boldsymbol{\omega}_{i} p_{F}+2 m p_{L}\right\|_{T^{1}}$ can be as small as one likes for very large $m$ so that the momenta of the field can be also very close to the singularity. However by the diophantine condition this will happen only for very large $m$, see Lemma 2.7 below, and using the exponential decay of $\hat{\varphi}_{m}$ we will see that these terms are indeed irrelevant.

The relevant terms are of two kinds; the $\nu$ terms, reflecting the renormalization of the Fermi momentum, and the $\sigma$ terms, related to the presence of a gap in the spectrum. The first kind of terms are faced in a standard way [BG] fixing properly the counterterm $\nu$ in the hamiltonian i.e. fixing properly the chemical potential. On the contrary there are no free parameters for the $\sigma$ terms in the hamiltonian and one has to proceed in a different way. One can naively think to perform a Bogolubov transformation, so considering as free propagator a propagator corresponding to a theory with a gap $O(u)$ at the Fermi surface. This is essentially what one does in the $\lambda=0$ case, see [BGM1], but here this does not work as the gap is deeply renormalized by the interaction and one has to perform different Bogolubov transformations at each integration. In fact $\sigma_{h}$ is a sort of "mass terms" with a non trivial renormalization group flow.

Regarding the marginal terms, there is an anomalous wave function renormalization which one has to take into account, what is expected as if $u=0$ the theory is a Luttinger liquid. In general the flow of the marginal terms can be controlled using some cancellations due to the fact that the Beta function is "close" (for small $u$ ) to the Luttinger model Beta function. In lemma 2.4 we write the propagator as the Luttinger model propagator plus a remainder, so that the Beta function is equal to the Luttinger model Beta function plus a "remainder" which is small if $\sigma_{h} \gamma^{-h}$ is small.

2.7 LEMMA Assume that $\sum_{i=1}^{n} \sigma_{i} \omega_{i} p_{F}+2 m p_{L} \neq 0$ mod. $2 \pi$. Then the contributions to $\mathcal{V}^{(h)}$ of the form (2.46) are vanishing unless

$$
|m| \geq \mathcal{A}\left[\frac{\gamma^{\frac{-h}{\tau}}}{n^{1 / \tau}}-\bar{m} n\right]
$$

if $\mathcal{A}$ is a suitable positive constant

PROOF Remembering the compact support of the Grassmanian operator $\psi_{\mathbf{k}_{i}^{\prime}+\omega_{i} \mathbf{p}_{F}, \omega_{i}}^{\sigma_{i}(\leq h)}$ we can write, using (2.46)

$$
a_{0} n \gamma^{h} \geq\left\|\sum_{i=1}^{n} \sigma_{i} k_{i}^{\prime}\right\|_{T^{1}} \geq\left\|2 m p_{L}+\sum_{i=1}^{n} \sigma_{i} \omega_{i} p_{F}\right\|_{T^{1}} \geq C_{0}(2|n| \bar{m}+m)^{-\tau}
$$

from which (2.47) follows.

Let be

$$
h^{*}=\inf \left\{h \geq h_{\beta}: a_{0} \gamma^{h+1} \geq 4\left|\sigma_{h}\right|\right\}
$$

From Lemma 2.4 it follows trivially: 
2.8 LEMMA For $h>h^{*}$ for any integer $N>1$ it is possible to find a $C_{N}$ such that, for $|x-y| \leq \frac{L}{2},\left|x_{0}-y_{0}\right| \leq \frac{\beta}{2}$

$$
\left|g_{\omega, \omega^{\prime}}^{(h)}(\mathbf{x} ; \mathbf{y})\right| \leq \frac{C_{N}\left(\max \left\{\gamma^{h}, L^{-1}\right\}\right)}{1+\left(\gamma^{h}|\mathbf{x}-\mathbf{y}|\right)^{N}}
$$

Proof For any $N \geq 0$, there is a constant $G_{N}$ such that, if $0 \geq h \geq h^{*}, N_{0}, N_{1} \geq 0$ and $N_{0}+N_{1}=N$,

$$
\left|D_{0}^{N_{0}} D_{1}^{N_{1}} \tilde{g}_{\omega, \omega^{\prime}}^{(h)}\left(\mathbf{k}^{\prime}\right)\right| \leq G_{N} \gamma^{-h N} \frac{\max \left\{\gamma^{h},\left|\sigma_{h}\right|\right\}}{\gamma^{2 h}+\sigma_{h}^{2}},
$$

where $D_{0}$ and $D_{1}$ denote the discrete derivative with respect to $k_{0}$ and $k^{\prime}$, respectively.

Hence, if $\left|x_{0}-y_{0}\right| \leq \beta / 2$ and $|x-y| \leq L_{i} / 2$, we have

$$
\begin{aligned}
& \left(\frac{\sqrt{2}}{\pi}\right)^{N}\left|x_{0}-y_{0}\right|^{N_{0}}|x-y|^{N_{1}}\left|g^{(h)}(\mathbf{x} ; \mathbf{y})\right| \leq \\
& \leq\left|\frac{\beta}{2 \pi}\left[e^{-i \frac{2 \pi}{\beta}\left(x_{0}-y_{0}\right)}-1\right]\right|^{N_{0}}\left|\frac{L_{i}}{2 \pi}\left[e^{-i \frac{2 \pi}{L_{i}}(x-y)}-1\right]\right|^{N_{1}}\left|g^{(h)}(\mathbf{x} ; \mathbf{y})\right|= \\
& =\left|\sum_{\omega, \omega^{\prime}= \pm 1} e^{-i\left(\omega x-\omega^{\prime} y\right) p_{F}} \frac{1}{L_{i} \beta} \sum_{\mathbf{k} \in \mathcal{D}_{L_{i}, \beta}} e^{-i \mathbf{k}^{\prime} \cdot(\mathbf{x}-\mathbf{y})} D_{0}^{N_{0}} D_{1}^{N_{1}} \tilde{g}_{\omega, \omega^{\prime}}^{(h)}\left(\mathbf{k}^{\prime}\right)\right| \leq \\
& \leq C_{N} \gamma^{h}\left(\max \left\{\gamma^{h}, L^{-1}\right\}\right) \gamma^{-h N} \frac{\max \left\{\gamma^{h},\left|\sigma_{h}\right|\right\}}{\gamma^{2 h}+\sigma_{h}^{2}} \leq C_{N} \gamma^{-h N}\left(\max \left\{\gamma^{h}, L^{-1}\right\}\right),
\end{aligned}
$$

where $C_{N}$ denotes a varying constant, depending only on $N$, and the factor $\left(\max \left\{\gamma^{h}, L^{-1}\right\}\right)$ arises from the sum over $\mathbf{k}^{\prime}$ (note that the sum over $k_{0}$ always gives a factor $\gamma^{h}$, since $\left.h_{\beta} \leq h^{*} \leq h\right)$. Therefore we have

$$
\left|g^{(h)}(\mathbf{x} ; \mathbf{y})\right| \leq \frac{C_{N} \max \left\{\gamma^{h}, L^{-1}\right\}}{1+\gamma^{h N}|\mathbf{x}-\mathbf{y}|^{N}}
$$

2.9 In the next section we will see that, using the above lemmas and assuming that the running coupling constants are bounded, the integrations (2.24) are well defined for $0 \geq h>$ $h^{*}$.

The integration of the scale from $h^{*}$ to $h_{\beta}$ can be performed "in a single step"

$$
\left.\int P_{Z_{h^{*}}}\left(d \psi^{\left(\leq h^{*}\right)}\right) e^{-\mathcal{V}^{h^{*}}\left(\sqrt{Z_{h^{*}}} \psi^{\left(\leq h^{*}\right)}\right.}=\frac{1}{\mathcal{N}_{h^{*}}} \int \tilde{P}_{Z_{h^{*}-1}}\left(d \psi^{\left(\leq h^{*}\right)}\right) e^{-\tilde{\mathcal{V}}^{h^{*}}\left(\sqrt{Z_{h^{*}}}\left(\leq h^{*}\right)\right.}\right)
$$

Calling $\int \tilde{P}_{Z_{h^{*}-1}}\left(d \psi^{\left(\leq h^{*}\right)}\right) \psi_{\mathbf{x}}^{+\left(\leq h^{*}\right)} \psi_{\mathbf{y}}^{-\left(\leq h^{*}\right)} \equiv \frac{g^{\left(\leq h^{*}\right)}(\mathbf{x} ; \mathbf{y})}{Z_{h^{*}-1}}$, we prove in the next section that the integration in the r.h.s. in (2.52) is well defined. This will be proved by using the following lemma (whose proof is similar to the proof of lemma 2.8).

2.10LEMMA Assume that $h^{*}$ is finite uniformly in $L, \beta$ so that $\left|\frac{\sigma_{h^{*}-1}}{\gamma^{*}}\right| \geq \bar{\kappa}$, if $\bar{\kappa}$ is a constant. Then it is possible to find a constant $C_{N}$ such that, for $|x-y| \leq \frac{L}{2},\left|x_{0}-y_{0}\right| \leq \frac{\beta}{2}$

$$
\left|g_{\omega, \omega^{\prime}}^{\left(\leq h^{*}\right)}(\mathbf{x} ; \mathbf{y})\right| \leq \frac{C_{N}\left(\max \left\{\gamma^{h^{*}}, L^{-1}\right\}\right)}{1+\left(\gamma^{h^{*}}|\mathbf{x}-\mathbf{y}|\right)^{N}}
$$

2.11 From Lemma 2.7 we see that it is possible to have quartic or bilinear contribution to $\mathcal{V}^{(h)}$, if $|h|$ is large enough, with $\sum_{i} \sigma_{i} \boldsymbol{\omega}_{i} p_{F}+2 m p_{L}=0$ mod. $2 \pi$ only with a extremely 
large $m$, namely $|m| \geq C \gamma^{\frac{-h}{\tau}}$; to show that such terms are irrelevant we use the fact that $\left|\hat{\phi}_{m}\right| \leq C e^{-\xi|m|}$, see 3.6. Comparing Lemma 2.8 and Lemma 2.10 we see that the propagator of the integration of all the scale between $h^{*}$ and $h_{\beta}$ has the same bound as the propagator of the integration of a single scale greater than $h^{*}$; this will be used to perform the integration of all the scales $\leq h^{*}$ in a single step. In fact $\gamma^{h^{*}}$ is a momentum scale and, roughly speaking, for momenta bigger than $\gamma^{h^{*}}$ the theory is "essentially" a massless theory (up to $O\left(\sigma_{h} \gamma^{-h}\right)$ terms) while for momenta smaller than $\gamma^{h^{*}}$ is a "massive" theory with mass $O\left(\gamma^{h^{*}}\right)$.

\section{Analiticity of the effective potential}

3.1 We find convenient in order to discuss the convergence of the expansion for the effective potential $\mathcal{V}^{(h)}$ to pass to the coordinate representation.

Let be

$$
\psi_{\mathbf{x}, \omega}^{(\leq h) \sigma}=\frac{1}{L \beta} \sum_{\mathbf{k}^{\prime} \in \mathcal{D}_{L, \beta}} e^{i \sigma \mathbf{k}^{\prime} \mathbf{x}} \psi_{\mathbf{k}^{\prime}+\omega \mathbf{p}_{F}, \omega}^{(\leq h) \sigma}
$$

and by (2.26) (2.27) we have, if $\mathcal{R}=1-\mathcal{L}$ :

1)for $n>4$

$$
\begin{gathered}
\sum_{x_{1}, \ldots, x_{n} \in \Lambda} \int_{-\beta / 2}^{\beta / 2} d x_{0,1} \ldots d x_{0, n} \mathcal{R}\left\{\prod_{i=1}^{n} \psi_{\mathbf{x}_{i}, \omega_{i}}^{(\leq h) \sigma_{i}} W_{n, m}^{h}\right\}= \\
\sum_{x_{1}, \ldots, x_{n} \in \Lambda} \int_{-\beta / 2}^{\beta / 2} d x_{0,1} \ldots d x_{0, n} \prod_{i=1}^{n} \psi_{\mathbf{x}_{i}, \omega_{i}}^{(\leq h) \sigma_{i}} W_{n, m}^{h}
\end{gathered}
$$

2)for $n=4$ and $\sum_{i=1}^{4} \sigma_{i} \omega_{i} p_{F}+2 m p_{L} \neq 0 \bmod .2 \pi$ one has $\mathcal{R}=I$ otherwise

$$
\begin{gathered}
\sum_{x_{1}, \ldots, x_{4} \in \Lambda} \int_{-\beta / 2}^{\beta / 2} d x_{0,1} \ldots d x_{0,4} \prod_{i=1}^{4} \psi_{\mathbf{x}_{i}, \omega_{i}}^{(\leq h) \sigma_{i}} \mathcal{R}\left\{W_{4, m}^{h}\left(\mathbf{x}_{1}-\mathbf{x}_{4}, \mathbf{x}_{2}-\mathbf{x}_{4}, \mathbf{x}_{3}-\mathbf{x}_{4} ; x_{4} ;\{\omega\}\right)\right\}=(3.1) \\
\quad \sum_{x_{1}, \ldots, x_{4} \in \Lambda} \int_{-\beta / 2}^{\beta / 2} d x_{0,1} \ldots d x_{0,4} \prod_{i=1}^{4} \psi_{\mathbf{x}_{i}, \omega_{i}}^{(\leq h) \sigma_{i}}\left[W_{4, m}^{h}\left(\mathbf{x}_{1}-\mathbf{x}_{4}, \mathbf{x}_{2}-\mathbf{x}_{4}, \mathbf{x}_{3}-\mathbf{x}_{4} ; x_{4} ;\{\omega\}\right)-\right. \\
\left.-\delta\left(x_{0,1}-x_{0,2}\right) \delta\left(x_{0,2}-x_{0,3}\right) \delta\left(x_{0,3}-x_{0,4}\right) \delta_{x_{1}, x_{2}} \delta_{x_{2}, x_{3}} \delta_{x_{3}, x_{4}} \sum_{\mathbf{t}_{1}, \mathbf{t}_{2}, \mathbf{t}_{3} \in \Lambda} W_{4, m}^{h}\left(\mathbf{t}_{1}, \mathbf{t}_{2}, \mathbf{t}_{3} ;\{\omega\}\right)\right]
\end{gathered}
$$

where we have used that if $\sum_{i=1}^{4} \sigma_{i} \omega_{i} p_{F}+2 m p_{L}=0 \bmod .2 \pi$ the kernels $W_{4, m}^{h}$ are translation invariant.

3)for $n=2$ and $\sum_{i=1}^{2} \sigma_{i} \omega_{i} p_{F}+2 m p_{L} \neq 0$ mod. $2 \pi$ then $\mathcal{R}=I$ otherwise

$$
\begin{gathered}
\sum_{x_{1}, x_{2} \in \Lambda} \int_{-\beta / 2}^{\beta / 2} d x_{0,1} d x_{0,2} \mathcal{R}\left\{\prod_{i=1}^{2} \psi_{\mathbf{x}_{i}, \omega_{i}}^{(\leq h) \sigma_{i}} W_{2, m}^{h}\left(\mathbf{x}_{1}-\mathbf{x}_{2} ; x_{2} ;\{\omega\}\right)\right\}= \\
\sum_{x_{1}, x_{2} \in \Lambda} \int_{-\beta / 2}^{\beta / 2} d x_{0,1} d x_{0,2} \prod_{i=1}^{2} \psi_{\mathbf{x}_{i}, \omega_{i}}^{(\leq h) \sigma_{i}}\left[W_{2, m}^{h}\left(\mathbf{x}_{1}-\mathbf{x}_{2} ; x_{2} ;\{\omega\}\right)-\right. \\
\delta\left(x_{0,1}-x_{0,2}\right) \delta_{x_{1}, x_{2}} \sum_{\mathbf{t} \in \Lambda} W_{2, m}^{h}(\mathbf{t} ;\{\omega\})+
\end{gathered}
$$




$$
\begin{aligned}
\partial_{x_{2}^{0}} \delta\left(x_{0,1}-x_{0,2}\right) \delta_{x_{1}, x_{2}} & \sum_{\mathbf{t} \in \Lambda} t_{0} W_{2, m}^{h}(\mathbf{t} ;\{\omega\})+\delta\left(x_{0,1}-x_{0,2}\right) \partial_{x_{2}} \delta_{x_{1}, x_{2}} \sum_{\mathbf{t} \in \Lambda} t W_{2, m}^{h}(\mathbf{t} ;\{\omega\})+ \\
& \left.\omega_{1} \delta\left(x_{0,1}-x_{0,2}\right) \delta_{x_{1}, x_{2}}^{2} \sum_{\mathbf{t} \in \Lambda} t W_{2, m}^{h}(\mathbf{t} ;\{\omega\})\right]
\end{aligned}
$$

and again we have used that when the $\mathcal{R}$ operation acts non trivially the kernels are translation invariant; moreover $\partial_{x_{2}} \delta_{x_{1}, x_{2}}=\frac{1}{2}\left[\delta_{x_{1}, x_{2}+1}-\delta_{x_{1}, x_{2}-1}\right]$ and $\partial_{x_{2}}^{2} \delta_{x_{1}, x_{2}}=\frac{i \cos p_{F}}{2}\left[\delta_{x_{1}, x_{2}+1}+\right.$ $\left.\delta_{x_{1}, x_{2}-1}-2 \delta_{x_{1}, x_{2}}\right]$.

The following identities, obtained by (3.1) (3.2), will be useful in the following

$$
\begin{gathered}
\sum_{\mathbf{x}_{1}, \ldots, \mathbf{x}_{4} \in \Lambda} \int_{\frac{-\beta}{2}}^{\frac{\beta}{2}} d x_{0,1} \ldots d x_{0,4}\left(\mathbf{x}_{i}-\mathbf{x}_{j}\right)^{a} \mathcal{R}\left\{\prod_{i=1}^{4} \psi_{\mathbf{x}_{i}, \omega_{i}}^{(\leq h) \sigma_{i}} W_{4, m}^{h}\left(\mathbf{x}_{1}-\mathbf{x}_{4}, \mathbf{x}_{2}-\mathbf{x}_{4}, \mathbf{x}_{3}-\mathbf{x}_{4} ; x_{4} ;\{\omega\}\right)\right\}= \\
\sum_{x_{1}, \ldots, x_{4} \in \Lambda} \int_{\frac{\beta}{2}}^{\frac{\beta}{2}} d x_{0,1} \ldots d x_{0,4}\left(\mathbf{x}_{i}-\mathbf{x}_{j}\right)^{a} \prod_{i=1}^{4} \psi_{\mathbf{x}_{i}, \omega_{i}}^{(\leq h) \sigma_{i}} W_{4, m}^{h}\left(\mathbf{x}_{1}-\mathbf{x}_{4}, \mathbf{x}_{2}-\mathbf{x}_{4}, \mathbf{x}_{3}-\mathbf{x}_{4} ; x_{4} ;\{\omega\}\right)
\end{gathered}
$$

if $a$ is any integer $\geq 1$ and $i, j$ can take any value between 1 and 4 .

$$
\begin{gathered}
\sum_{x_{1}, x_{2} \in \Lambda} \int_{\frac{-\beta}{2}}^{\frac{\beta}{2}} d x_{0,1} d x_{0,2}\left(x_{1}-x_{2}\right)^{a} \mathcal{R}\left\{\prod_{i=1}^{2} \psi_{\mathbf{x}_{i}, \omega_{i}}^{(\leq h) \sigma_{i}} W_{2, m}^{h}\left(\mathbf{x}_{1}-\mathbf{x}_{2} ; x_{2} ;\{\omega\}\right)\right\}= \\
\sum_{x_{1}, x_{2} \in \Lambda} \int_{\frac{-\beta}{2}}^{\frac{\beta}{2}} d x_{0,1} d x_{0,2}\left[\prod_{i=1}^{2} \psi_{\mathbf{x}_{i}, \omega_{i}}^{(\leq h) \sigma_{i}}\left(x_{1}-x_{2}\right)^{a} W_{2,}^{h}\left(\mathbf{x}_{1}-\mathbf{x}_{2} ; x_{2} ;\{\omega\}\right)-\right. \\
\frac{1}{2} \psi_{\mathbf{x}_{1}}^{\sigma_{1}(\leq h)}\left[\psi_{x_{1}+1, x_{0,1}}^{\sigma_{2}(\leq h)}(-1)^{a}-\psi_{x_{1}-1, x_{0,1}}^{\sigma_{2}(\leq h)}\right]\left(x_{1}-x_{2}\right) W_{2, m}^{h}\left(\mathbf{x}_{1}-\mathbf{x}_{2} ; x_{2} ;\{\boldsymbol{\omega}\}\right)+ \\
\left.+\frac{i \omega}{2} \cos \left(p_{F}\right) \psi_{\mathbf{x}_{1}}^{\sigma_{1}(\leq h)}\left[\psi_{x_{1}-1, x_{0,1}}^{\sigma_{2}(\leq h)}+(-1)^{a} \psi_{x_{1}+1, x_{0,1}}^{\sigma_{2}(\leq h)}\right]\left(x_{1}-x_{2}\right) W_{2, m}^{h}\left(\mathbf{x}_{1}-\mathbf{x}_{2} ; x_{2} ;\{\boldsymbol{\omega}\}\right)\right]
\end{gathered}
$$

if $a \geq 1$ and a similar equation hold if $\left(x_{1}-x_{2}\right)$ is replaced by $\left(x_{0,1}-x_{0,2}\right)$.

Of course one can integrate the $\delta$ functions in (3.1) (3.2) so obtaining

$$
\begin{gathered}
\sum_{x_{1}, \ldots, 4 \in \Lambda} \int_{\frac{-\beta}{2}}^{\frac{\beta}{2}} d x_{0,1} \ldots d x_{0,4} \mathcal{R}\left\{\prod_{i=1}^{4} \psi_{\mathbf{x}_{1}, \omega_{i}}^{(\leq h) \sigma_{i}} W_{4, m}^{h}\left(\mathbf{x}_{1}-\mathbf{x}_{4}, \mathbf{x}_{2}-\mathbf{x}_{4}, \mathbf{x}_{3}-\mathbf{x}_{4} ; x_{4} ;\{\omega\}\right)\right\}= \\
\sum_{x_{1}, \ldots, 4 \in \Lambda} \int_{\frac{-\beta}{2}}^{\frac{\beta}{2}} W_{4, m}^{h}\left(\mathbf{x}_{1}-\mathbf{x}_{2}, \mathbf{x}_{2}-\mathbf{x}_{4}, \mathbf{x}_{3}-\mathbf{x}_{4} ; x_{4} ;\{\omega\}\right) \\
{\left[\psi_{\mathbf{x}_{1}, \omega_{1}}^{(\leq h) \sigma_{1}} \psi_{\mathbf{x}_{2}, \omega_{2}}^{(\leq h) \sigma_{2}} \psi_{\mathbf{x}_{3}, \omega_{3}}^{(\leq h) \sigma_{3}} \psi_{\mathbf{x}_{4}, \omega_{4}}^{(\leq h) \sigma_{4}}-\psi_{\mathbf{x}_{1}, \omega_{1}}^{(\leq h) \sigma_{1}} \psi_{\mathbf{x}_{1}, \omega_{2}}^{(\leq h) \sigma_{2}} \psi_{\mathbf{x}_{1}, \omega_{3}}^{(\leq h) \sigma_{3}} \psi_{\mathbf{x}_{1}, \omega_{4}}^{(\leq h) \sigma_{4}}\right]}
\end{gathered}
$$

The square brakets in the above equation can be written as

$$
\begin{gathered}
\psi_{\mathbf{x}_{1}, \omega_{1}}^{(\leq h) \sigma_{1}} D_{\mathbf{x}_{2,1}, \omega_{2}}^{(\leq h) \sigma_{2}} \psi_{\mathbf{x}_{3}, \omega_{3}}^{(\leq h) \sigma_{2}} \psi_{\mathbf{x}_{4}, \omega_{4}}^{(\leq h) \sigma_{4}} \\
+\psi_{\mathbf{x}_{1}, \omega_{1}}^{(\leq h) \sigma_{1}} \psi_{\mathbf{x}_{1}, \omega_{2}}^{(\leq h) \sigma_{2}} D_{\mathbf{x}_{3,1}, \omega_{3}}^{(\leq h) \sigma_{3}} \psi_{\mathbf{x}_{4}, \omega_{4}}^{(\leq h) \sigma_{4}}+\psi_{\mathbf{x}_{1}, \omega_{1}}^{(\leq h) \sigma_{1}} \psi_{\mathbf{x}_{1}, \omega_{2}}^{(\leq h) \sigma_{2}} \psi_{\mathbf{x}_{1}, \omega_{3}}^{(\leq h) \sigma_{3}} D_{\mathbf{x}_{1,4}, \omega_{4}}^{(\leq h) \sigma_{4}}
\end{gathered}
$$

so that the effect of $\mathcal{R}$ is essentially to change the coordinate of the $\psi_{\mathbf{x}_{i}, \omega}^{(\leq h) \sigma_{i}}$ fields and to replace a field $\psi_{\mathbf{x}_{i}, \omega}^{(\leq h) \sigma_{i}}$ with $D_{\mathbf{x}_{i j}}^{(\leq h) \sigma_{i}}$ where

$$
D_{\mathbf{x}_{i j}}^{(\leq h) \sigma_{i}}=\psi_{\mathbf{x}_{i}, \omega_{i}}^{(\leq h) \sigma_{i}}-\psi_{\mathbf{x}_{j}, \omega_{i}}^{(\leq h) \sigma_{i}}=\left(\mathbf{x}_{i}-\mathbf{x}_{j}\right) \int_{0}^{1} d t \vec{\partial} \psi_{\mathbf{x}_{j i}(t), \omega_{i}}^{(\leq h) \sigma_{i}}
$$


with $\mathbf{x}_{j i}(t)=t \mathbf{x}_{j}+(1-t) \mathbf{x}_{i}$ and $\vec{\partial}=\left(\partial_{x}, \partial_{x_{0}}\right)$. In the same way integrating the $\delta$ 's in (3.2) one can see that the effect of $\mathcal{R}$ is to replace a field $\psi^{(\leq h) \sigma}$ with a field $A^{(\leq h) \sigma}$ which can be written as:

$$
\left(\mathbf{x}_{2}-\mathbf{x}_{1}\right)^{2} \int_{0}^{1} d t_{1} \int_{0}^{t_{1}} d t_{2} \partial^{2} \psi_{x_{21}\left(t_{2}\right)}^{(\leq h) \sigma}+\left(x_{2}-x_{1}\right) \Delta \psi_{x_{2}}^{(\leq h) \sigma}
$$

where $\Delta \psi_{x_{2}}^{(\leq h) \sigma}$ is defined implicitely by the above equation and it has the same scaling properties of $\partial^{2} \psi_{x_{2}}^{(\leq h) \sigma}$. The $t$-parameters appearing in (3.6)(3.7) are called interpolation parameters.

3.2 From (3.5),(3.6) one can see that the effect of $\mathcal{R}$ is that a derivative is applied on a field and there is a factor $\left(\mathbf{x}_{i}-\mathbf{x}_{j}\right)$ more in the kernels. With respect to the $\mathcal{R}=1$ case the presence of the derivative produce a gain in the bounds and the factor $\left(\mathbf{x}_{i}-\mathbf{x}_{j}\right)$ a loss (we are studying the large distance behaviour) and at the end, due to the structure of the iterative integration discussed in sec.2, the final effect is a gain. This is standard in renormalization group formalism, see [G2]. Analogous considerations can be done for (3.7).

From (3.3) we see that, by definition, $\mathcal{R}$ does not act if there is a factor $\left(\mathbf{x}_{i}-\mathbf{x}_{j}\right)^{a}$ in front of $\mathcal{R} W_{4, m}^{h}$. This property will simplify the discussion and was already used in [BM1], if the fermions are on the continuum and not on a chain; (3.4) is the analogue for the $n=2$ case. Note the two different equivalent ways to write the $\mathcal{R}$ operation: in (3.1),(3.2) the $\mathcal{R}$ acts on the kernels of the effective potential; we can say that it consists in replacing $W_{n, m}^{h}$ with $\mathcal{R} W_{n, m}^{h}$ where $\mathcal{R} W_{n, m}^{h}$ are the terms in square brakets in r.h.s of (3.1),(3.2), leaving the fermionic fields unchanged; on the other hand writing as in (3.5) the $\mathcal{R}$ operation acts on the $\psi$ fields, leaving the kernel $W_{n, m}^{h}$ unchanged.

3.3 From (2.43) we have that, if $E_{h+1}^{T}$ denotes the truncated expectation with propagator $\frac{g^{(h+1)}(\mathbf{x} ; \mathbf{y})}{Z_{h}}(2.33)$ :

$$
\mathcal{V}^{h}\left(\sqrt{Z_{h}} \psi^{\leq h}\right)=\sum_{n=1}^{\infty} \frac{1}{n !}(-1)^{n+1} E_{h+1}^{T}\left(\hat{\mathcal{V}}^{h+1}\left(\sqrt{Z_{h}} \psi \leq h+1\right), \ldots\right)
$$

where $\hat{\mathcal{V}}^{h+1}$ is obtained from $\mathcal{V}^{h+1}$ following the operations described in sec.2. Iterating the above equation we obtain that the effective potential can be written in term of a tree expansion [G2].

A tree $\tau$ consists of a family of lines arranged to connect a partially ordered set of points (vertices). Every line has two vertices at the extreme except the first one which has only one vertex, the first vertex $v_{0}$. The other extreme $r$ is the root and it is not a vertex. From each vertex $v$ start $s_{v}$ lines; if $s_{v}=0$ the vertex is called end-point, if $s_{v}=1$ is called trivial vertex and if $s_{v}>1$ is called non trivial vertex. To each vertex $v$ we associate a scale $h_{v}$. The vertices of $\tau$ are naturally (partially) ordered. Giving to the tree an orientation from left to right we write $v_{1}<v_{2}$ (so that $h_{v_{1}}<h_{v_{2}}$ ) if $v_{1}$ is before $v_{2}$ on the tree. Two trees that can be superposed by a suitable continuous deformation are equivalent i.e. the trees 
are topological trees. The number of trees with $n$ end-points is bounded by $C^{n}$, if $C$ is a constant.

To each end point $v$ with scale $h_{v}$ we associate either one of the addend of $\mathcal{L} V^{\left(h_{v}-1\right)}$ in (2.42) or one of the terms of $\mathcal{R} V^{0}$; this second case is possible only if the scale of the end point is 1 . For simplicity of notations we assume in this section

$$
V^{0}=\sum_{m \neq 0, \pm \bar{m}} e^{i 2 m p_{L} x} \sum_{x \in \Lambda} \int_{-\beta / 2}^{\frac{\beta}{2}} d x_{0} \hat{\phi}_{m} \psi_{\mathbf{x}}^{(\leq h)+} \psi_{\mathbf{x}}^{(\leq h)-}
$$

It will be clear from the following consideration that the general case is completely equivalent. Between two non trivial vertices $v_{1}, v_{2}$ there are all the trivial vertices $v$ with scales $h_{v_{1}}<$ $h_{v}<h_{v_{2}}$. If $n \geq 2$ and the scale of an end point is different from 1 there is no trivial vertex between the end-point and the non trivial vertex $v$ immediately preceding it on the tree, so that the scale of the end point is $h_{v}+1$ and the running coupling constants associated to it is one of the $\vec{v}_{h_{v}}$. If $n=1$ and to the end point is associated a running coupling constant there is only a vertex $v_{0}$ on the tree, besides the end-point, and the scale of the running coupling constant is $h_{v_{0}}=h_{k+1}$. Each tree $\tau$ take a label distinguishing which term is associated to the end points. Each trivial or non trivial vertex carry a label $\mathcal{R}$ except $v_{0}$ which can carry an $\mathcal{R}$ or $\mathcal{L}$ operation. The set of all the trees with $n$ end points with root with scale $k$ will be denoted by $\tau_{n, k}$.

A cluster $L_{v}$ with frequeny $h_{v}$ is the set of the end points (possibly only one) reachable from the vertex $v$, and the tree provides an organization of end points into a hierarchy of clusters. Given a cluster $L_{v}$ (with scale $h_{v}$ ), we define

$$
N_{v}=\sum_{i \in L_{v}} m_{i}
$$

where the index $i$ denotes the end-points contained in $L_{v}, m_{i}=0$ if to the end-point $i$ is associated a running coupling constant not of the kind $\tau, \iota, m_{i}= \pm \bar{m}$ if to the end-point $i$ is associated a running coupling constant of the kind $\tau, \iota$ and finally if to the end point $i$ is associated one of the irrelevant terms $(3.8) m_{i} \neq 0, \pm \bar{m}$. By definition

$$
N_{v}=N_{v^{1}}+\ldots+N_{v^{s}}
$$

and if $v_{i}$ is an end point than $N_{v}=m_{i}$.

Following standard arguments (see [G2]) the effective potential can be written in the following way

$$
\mathcal{V}^{(k)}\left(\sqrt{Z_{k}} \psi^{(\leq k)}\right)=\sum_{n=1}^{\infty} \sum_{\tau \in \tau_{k, n}} V^{k}\left(\tau, \sqrt{Z_{k}} \psi^{(\leq k)}\right)
$$

If $v_{0}$ is the first vertex after the root, $\tau_{1}, . ., \tau_{s_{v_{0}}}$ are the subtrees starting after the vertex $v_{0}$, then $V^{k}\left(\tau, \sqrt{Z_{k}} \psi^{(\leq k)}\right)$ is defined inductively by the relation

$$
V^{k}\left(\tau, \sqrt{Z_{k}} \psi^{(\leq k)}\right)=\frac{1}{s_{v_{0}} !} E_{k+1}^{T}\left[\bar{V}^{k+1}\left(\tau_{1}, \sqrt{Z_{k}} \psi^{(\leq k+1)}\right), . ., \bar{V}^{k+1}\left(\tau_{s_{v_{0}}}, \sqrt{Z_{k}} \psi^{(\leq k+1)}\right)\right]
$$

where $\bar{V}^{k+1}\left(\tau_{i}, \sqrt{Z_{k}} \psi^{(\leq k+1)}\right)$

1)is equal to $\mathcal{R} \hat{V}^{k+1}\left(\tau_{i}, \sqrt{Z_{k}} \psi^{(\leq k+1)}\right)$ if the subtree $\tau_{i}$ is not trivial i.e. if the first vertex of $\tau_{i}$ is not an and-point,

2)if the first vertex of $\tau_{i}$ is an end-point $\bar{V}^{k+1}\left(\tau_{i}, \sqrt{Z_{k}} \psi^{(\leq k+1)}\right)$ is equal to one addend of $\mathcal{L} \hat{V}^{k+1}\left(\tau_{i}, \sqrt{Z_{k}} \psi \leq k+1\right)(2.42)$ or, if $k=-1$, to one term of $V^{0}\left(\tau_{i}, \psi \leq k+1\right)$. 
Let we assume that the effective potential can be written as:

$$
V^{k}\left(\tau, \sqrt{Z_{k}} \psi^{(\leq k)}\right)=\int d \mathbf{x}_{v_{0}} \sum_{P_{v_{0}}}{\sqrt{Z_{k}}}^{\left|P_{v_{0}}\right|} \tilde{\psi}^{(\leq k)}\left(P_{v_{0}}\right) W^{k}\left(\tau, P_{v_{0}}, \mathbf{x}_{v_{0}}\right)
$$

where $\int d \mathbf{x}_{v_{0}}=\prod_{f \in I_{v_{0}}} \sum_{x(f) \in \Lambda} \int_{-\beta / 2}^{\beta / 2} d x(f), P_{v_{0}}$ is a non empty set of $I_{v_{0}}$, the field labels associated with the end-points reachable from $v_{0}$ (i.e. all of them), $\left|P_{v_{0}}\right|$ is the number of elements of $P_{v_{0}}, \sum_{P_{v_{0}}}$ is the sum over such sets, $x_{v_{0}}$ is the set $\{x(f)\}, f \in I_{v_{0}}$ i.e. the set of the coordinates associated with the tree and:

$$
\tilde{\psi}^{(\leq k)}\left(P_{v_{0}}\right)=\prod_{f \in P_{v}} \psi_{\mathbf{x}(f), \omega(f)}^{(\leq k) \sigma(f)}
$$

The above assumption is proved by induction assuming that (3.13) holds also for the subtrees $\tau_{i}$ and using (3.12). In fact by the identity

$$
\tilde{\psi}^{(\leq k+1)}(P)=\sum_{Q \subset P} \tilde{\psi}^{(<k+1)}(Q) \tilde{\psi}^{(k+1)}(P / Q)
$$

we obtain

$$
\begin{gathered}
V^{k}\left(\tau, \sqrt{Z_{k}} \psi^{(\leq k)}\right)=\frac{1}{s_{v_{0}} !} \sum_{P_{v_{0}}}{\sqrt{Z_{k}}}^{\left|P_{v_{0}}\right|} \sum_{P_{v_{0}^{1}}, \ldots, P_{v_{0}}} \sum_{s_{v_{0}}} \sum_{v_{0}^{1}, \ldots, Q_{v_{0}} s_{v_{0}}} \\
\mathcal{E}_{k+1}^{T}\left[\int d \mathbf{x}_{v_{0}^{1}} \tilde{\psi}^{(k+1)}\left(P_{v_{0}^{1}} / Q_{v_{0}^{1}}\right) \bar{W}^{k+1}\left(\tau_{1}, P_{v_{0}^{1}}, \mathbf{x}_{v_{0}^{1}}\right), \ldots,\right. \\
\left.\int d \mathbf{x}_{v_{0}^{s} v_{0}} \tilde{\psi}^{(k+1)}\left(P_{v_{0}^{s} v_{0}} / Q_{v_{0}^{s} v_{0}}\right) \bar{W}^{k+1}\left(\tau_{s_{v_{0}}}, P_{v_{0}^{s} v_{0}}, \mathbf{x}_{v_{0}^{s} v_{0}}\right)\right] \tilde{\psi}^{(\leq k)}\left(P_{v_{0}}\right)
\end{gathered}
$$

where $\mathcal{E}_{h}^{T}$ denotes the truncated expectation with respect to the propagator $g^{(h)}(\mathbf{x} ; \mathbf{y})(2.35)$, $P_{v_{0}} \cup_{i} Q_{v_{0}^{i}}, Q_{v_{0}^{i}} \subset P_{v_{0}^{i}}, v_{0}^{i}$ is the first vertex of the subtree $\tau_{i}$, and $\bar{W}^{k+1}\left(\tau, P_{v_{0}^{i}}, \mathbf{x}_{v_{0}^{i}}\right)=$ $\mathcal{R} \bar{W}^{k+1}\left(\tau, P_{v_{0}^{i}}, \mathbf{x}_{v_{0}^{i}}\right)$ if $\tau_{i}$ is not an end-point, $\bar{W}^{k+1}\left(\tau, P_{v_{0}^{i}}, \mathbf{x}_{v_{0}^{i}}\right)=\vec{v}_{k+1}$ if it is an end-point but $k+1 \neq 0$ and if it is an end-point with $k+1=0$ is equal to $\vec{v}_{0}$ or to $\hat{\phi}_{m} e^{i 2 m p_{L} x}$. The above expression of course proves (3.13).

We prove the following theorem, if $\vec{v}_{h}$ are the running coupling constants in $(2.42)$

3.4THEOREM Let be $k>h^{*}$ given by (2.48). There exists a constant $\bar{\varepsilon}_{k}$ such that, if $\sup _{h>k}\left|\vec{v}_{h}\right| \leq \bar{\varepsilon}_{k}$ and $\sup _{h>k}\left|\frac{Z_{h}}{Z_{h-1}}\right| \leq e^{c_{1} \bar{\varepsilon}_{k}^{2}}$, chosen $\gamma^{\frac{1}{\tau}} / 2 \geq 1$ and $L, \beta \geq \gamma^{-h^{*}}$, then

$$
\left|\int d \mathbf{x}^{P_{v_{0}}} \sum_{\tau \in \tau_{n, k}}\right| W^{k}\left(\tau, P_{v_{0}}, \mathbf{x}^{P_{v_{0}}}\right)\left|\left(1+\gamma^{k} d\left(P_{v_{0}}\right)^{N}\right)\right| \leq L \beta \gamma^{-k D\left(P_{v_{0}}\right)}\left(C_{N} \bar{\varepsilon}_{k}\right)^{n} e^{\frac{-\gamma^{-k / \tau} c_{2} P_{P_{v}}}{2^{-k+3}}}
$$

where $s_{P_{v_{0}}}=1$ if $N_{v_{0}} \neq 0,\left|P_{v_{0}}\right|=2$ and 0 otherwise, $N$ is a positive integer, $\mathbf{x}^{P_{v}{ }^{0}}$ is the set of points associated to $P_{v_{0}}, c_{1}, c_{2}, C_{N}$ are constants (not depending on $\left.L, \beta\right), d\left(P_{v_{0}}\right)$ is the length of the shortest tree connecting the set of points $\mathbf{x}^{P_{v_{0}}}$, and

$$
D\left(P_{v_{0}}\right)=-2+\sum_{f \in P_{v_{0}}}\left(1 / 2+m_{f}\right)
$$

where $m_{f}$ is the order of the derivative applied to the fields of label $f$.

3.5 PROOF In order to write in an explicit form (3.12) it is convenient to start studying $\mathcal{R} \hat{V}^{h}$ :

$$
\mathcal{R} \hat{V}^{k}\left(\tau, \sqrt{Z_{k-1}} \psi^{(\leq k)}\right)=\frac{1}{s_{v_{0}} !} \sum_{P_{v_{0}}}\left(\sqrt{Z_{k-1}}\right)^{\left|P_{v_{0}}\right|} \mathcal{R}\left\{\sum_{P_{v_{0}^{1}}, \ldots, P_{v_{0} s}^{s}}{\sqrt{\frac{Z_{k}}{Z_{k-1}}}}^{\left|P_{v_{0}}\right|}\right.
$$




$$
\begin{gathered}
\sum_{Q_{v_{0}^{1}}, . . Q_{v_{0}} v_{v_{0}}} \mathcal{E}_{k+1}^{T}\left[\int d \mathbf{x}_{v_{0}^{1}} \tilde{\psi}^{(k+1)}\left(P_{v_{0}^{1}} \backslash Q_{v_{0}^{1}}\right) \bar{W}^{k+1}\left(\tau, P_{v_{0}^{1}}, \mathbf{x}_{v_{0}^{1}}\right), \ldots,\right. \\
\left.\left.\int d \mathbf{x}_{v_{0}^{s} v_{0}} \tilde{\psi}^{(k+1)}\left(P_{v_{0}^{s v_{0}}} \backslash Q_{v_{0}^{s v_{0}}}\right) \bar{W}^{k+1}\left(\tau, P_{v_{0}^{s}}, \mathbf{x}_{v_{0}^{s v_{0}}}\right)\right] \tilde{\psi}^{(\leq k)}\left(P_{v_{0}}\right)\right\}
\end{gathered}
$$

From (3.5)-(3.7) we know that the effect of $\mathcal{R}$ is simply to replace $\tilde{\psi}^{(\leq k)}\left(P_{v_{0}}\right)$ with

$$
\sum_{i, j}^{*}\left(\mathbf{x}_{i}-\mathbf{x}_{j}\right)^{a_{v_{0}}} \hat{\psi}^{\leq k}\left(P_{v_{0}}\right)
$$

(in the following $\sum_{i, j}^{*}$ will be omitted), where $\mathbf{x}_{i}, \mathbf{x}_{j}$ are two coordinates in $\mathbf{x}^{P_{v_{0}}}$ and

1) $a_{v_{0}}=0$ and $\hat{\psi}^{(\leq k)}\left(P_{v_{0}}\right)=\tilde{\psi}^{(\leq k)}\left(P_{v_{0}}\right)$ if $\left|P_{v_{0}}\right|>4$ or $\sum_{f \in P_{v_{0}}} \sigma(f) \boldsymbol{\omega}(f) p_{F}+2 N_{v_{0}} p_{L} \neq 0$ mod. $2 \pi$, see $(2.26),(2.27)$.

$$
\text { 2)if }\left|P_{v_{0}}\right|=4 \text { and } \sum_{f \in P_{v_{0}}} \sigma(f) \boldsymbol{\omega}(f) p_{F}+2 N_{v_{0}} p_{L}=0 \text { mod. } 2 \pi \text { then } a_{v_{0}}=1 \text { and } \hat{\psi}^{(\leq k)}\left(P_{v_{0}}\right)
$$
differs from $\tilde{\psi}^{(\leq k)}\left(P_{v_{0}}\right)$ because a field $\int_{0}^{1} d t \partial \psi_{x_{i j}(t)}^{(\leq k)}$ replaces a $\psi^{(\leq k)}$ field, and the remaning $\psi$ fields are applied on different coordinates among $\mathbf{x}^{P_{v_{0}}}$, see (3.5);

3)if $\left|P_{v_{0}}\right|=2$ and $\sum_{f \in P_{v_{0}}} \sigma(f) \boldsymbol{\omega}(f) p_{F}+2 N_{v_{0}} p_{L}=0 \bmod .2 \pi$ then $a_{v_{0}}=2$ and $\hat{\psi}^{(\leq k)}\left(P_{v_{0}}\right)$ differs from $\tilde{\psi}^{(\leq k)}\left(P_{v_{0}}\right)$ because a field $\int_{0}^{1} d t_{1} \int_{0}^{t_{1}} d t_{2} \partial^{2} \psi_{x_{i j}\left(t_{2}\right)}^{(\leq k)}$ or a $\frac{\Delta \psi^{(\leq k)}}{\left(x_{1}-x_{2}\right)}$ replaces a $\psi^{(\leq k)}$ field.

Let we write:

$$
\hat{\psi}^{(h)}(P)=\prod_{f \in P} \partial_{\mathbf{x}(f)}^{q(f)} \psi_{\mathbf{x}(f)}^{\sigma(f)(h)}
$$

where $q=0,1,2,3, \partial^{0}=1, \partial^{1}=\partial_{\mathbf{x}}, \partial_{\mathbf{x}}^{2}=\partial_{\mathbf{x}} \partial_{\mathbf{x}}, \partial^{3}=\frac{1}{\left(x_{1}-x_{2}\right)} \Delta$.

We remember the well known expansion of truncated expectation in term of interpolating parameters $s_{t}, t=1, . ., k-1$ :

$$
\mathcal{E}_{h}^{T}\left(\hat{\psi}^{(h)}\left(P_{1}\right), \ldots, \hat{\psi}^{(h)}\left(P_{k}\right)\right)=\sum_{T} \prod_{l \in T} \partial_{\mathbf{x}_{l}}^{q_{l}} \partial_{\mathbf{y}_{l}}^{q_{l}^{\prime}} g^{(h)}\left(\mathbf{x}_{l} ; \mathbf{y}_{l}\right) \int d P_{T}(s) \operatorname{det} G^{h, T}(s)
$$

where $T$ is a set of lines forming an anchored tree graph between the clusters of vertices from which the fields labeled with $P_{1}, . ., P_{k}$ emerge: this means that $T$ is a set of lines connecting two points in different clusters, which becomes a tree graph if one identifies all the points in the same cluster; if $l \in T \mathbf{x}_{l}, \mathbf{y}_{l}$ are the end-points of the line and are such that $\mathbf{x}_{l}=\mathbf{x}_{i j}$ or $\mathbf{y}_{l}=\mathbf{x}_{i^{\prime} j^{\prime}}$, where $\mathbf{x}_{i j}$ denotes the coordinate of the $i$-th field of the monomial $\tilde{\psi}\left(P_{j}\right)$. If $f$ is a field variable such that $\mathbf{x}(f) \equiv \mathbf{x}_{l} \equiv \mathbf{x}_{i j}$, we write $q(f) \equiv q_{l} \equiv q_{i j}$. In the same way if $\bar{f}$ is such that $\mathbf{x}(\bar{f}) \equiv \mathbf{y}_{l} \equiv \mathbf{x}_{i^{\prime} j^{\prime}}$ we say $q(\bar{f}) \equiv q_{l}^{\prime}$. $G^{h, T}(s)$ is a $(n-k+1) \times(n-k+1)$ matrix (if $n$ is the total number of fields), whose elements are $G_{j i j^{\prime} i^{\prime}}^{T}=S_{j j^{\prime}} \partial^{q_{i j}} \partial^{q_{i^{\prime} j^{\prime}}} g^{h}\left(x_{i j}-x_{i^{\prime} j^{\prime}}\right)$ with $x_{i j}-x_{i^{\prime} j^{\prime}}$ non belonging to $T, S_{j j^{\prime}}=\prod_{t=j}^{j^{\prime}-1} s_{t}$ and $d P_{T}(s)$ is a normalized measure which depends on $s_{t}, t=1, \ldots, k-1$ and $T$ (for the well known explicit formula of $d P_{T}$ and for its derivation, one can look for instance [BGPS]).

We write in an explicit way the action of $\mathcal{R}$ in (3.15) obtaining

$$
\begin{gathered}
\mathcal{R} \hat{V}^{k}\left(\tau, \sqrt{Z_{k-1}} \psi^{(\leq k)}\right)=\frac{1}{s_{v_{0}} !} \sum_{P_{v_{0}}} \sqrt{Z_{k-1}}\left|P_{v_{0}}\right| \sum_{P_{v_{0}^{1}}, \ldots, P_{v_{0}}} \sqrt{\frac{Z_{k}}{Z_{k-1}}}{ }^{\left|P_{v_{0}}\right|} \\
\sum_{Q_{v_{0}^{1}}, . ., Q_{v_{0}} s_{v_{0}}} \int d \mathbf{x}_{v_{0}}\left[\left(\mathbf{x}_{i}-\mathbf{y}_{j}\right)^{a_{v_{0}}} \mathcal{E}_{k+1}^{T}\left(\tilde{\psi}^{(k+1)}\left(P_{v_{0}^{1}} \backslash Q_{v_{0}^{1}}\right), \ldots, \tilde{\psi}^{(k+1)}\left(P_{v_{0}} \backslash Q_{v_{0}} s_{v_{0}}\right)\right)\right]
\end{gathered}
$$




$$
\bar{W}^{k+1}\left(\tau_{1}, P_{v_{0}^{1}}, \mathbf{x}_{v_{0}^{1}}\right) \ldots \bar{W}^{k+1}\left(\tau_{s_{v_{0}}}, P_{v_{0}^{s_{v_{0}}}}, \mathbf{x}_{v_{0}}^{s_{v_{0}}}\right) \hat{\psi}^{(\leq k)}\left(P_{v_{0}}\right)
$$

We can write, for any anchored tree graph $T_{v_{0}}$ connecting the clusters $L_{v_{0}^{1}} \ldots L_{v_{0}}^{s_{v_{0}}}$ :

$$
\left(\mathbf{x}_{i}-\mathbf{y}_{j}\right)=\sum_{l \in T}\left(\mathbf{x}_{l}-\mathbf{y}_{l}\right)+\sum_{i j i^{\prime} j^{\prime}}^{*}\left(\mathbf{x}_{i j}-\mathbf{y}_{i^{\prime} j^{\prime}}\right)
$$

where $\sum_{i j i^{\prime} j^{\prime}}^{*}$ is a sum such that there is no $l \in T_{v_{0}}$ such that $\mathbf{x}_{i j}=\mathbf{x}_{l}, \mathbf{y}_{i^{\prime} j^{\prime}}=\mathbf{y}_{l}$.

So we can write:

$$
\begin{gathered}
\left(\mathbf{x}_{i}-\mathbf{y}_{i}\right)^{a_{v_{0}}} \mathcal{E}_{k+1}^{T}\left(\tilde{\psi}^{(k+1)}\left(P_{v_{0}^{1}} \backslash Q_{v_{0}^{1}}\right), \ldots\right) \bar{W}^{k+1}\left(\tau_{1}, P_{v_{0}^{1}}, \mathbf{x}_{v_{0}^{1}}\right) \ldots \bar{W}^{k+1}\left(\tau_{s_{v_{0}}}, P_{v_{0}} s_{v_{0}}, \mathbf{x}_{v_{0}}{ }^{s_{v_{0}}}\right) \\
=\sum_{T_{v_{0}}} \sum_{\{a\}}^{*}\left\{\left[\prod_{l \in T_{v_{0}}}\left(\mathbf{x}_{l}-\mathbf{y}_{l}\right)^{a_{l}} g^{(k+1)}\left(\mathbf{x}_{l} ; \mathbf{y}_{l}\right)\right]\left[\int d P_{\left.T_{v}\right)}(s) \operatorname{det} G^{k+1, T_{v_{0}}}(s)\right]\right. \\
\left.\left|\hat{\mathbf{x}}_{v_{0}^{1}}\right|^{a_{v_{0}^{1}}} \bar{W}^{k+1}\left(\tau_{1}, P_{v_{0}^{1}}, \mathbf{x}_{v_{0}^{1}}\right) \ldots\left|\hat{\mathbf{x}}_{v_{0}^{s}}{ }^{s_{v_{0}}}\right|^{a v_{0}^{s_{0}}} \bar{W}^{k+1}\left(\tau_{s_{v_{0}}}, P_{v_{0}}^{s_{v_{0}}}, \mathbf{x}_{v_{0}^{s_{v_{0}}}}\right)\right\}
\end{gathered}
$$

where $\left|\hat{\mathbf{x}}_{v_{0}^{i}}\right|$ is the difference among two coordinates in $\mathbf{x}_{P^{v_{0}^{i}}}$, and $\sum_{\{a\}}^{*}$ is the sum over the indices $a_{l}+a_{v_{0}^{1}}+.$. with the constraint that $\sum_{l \in T_{v_{0}}} a_{l}+a_{v_{0}^{1}}+\ldots+a_{v_{0}^{s}}=a_{v_{0}} \leq 2$. We are now in position to iterate the above procedure studying each $\left|\hat{\mathbf{x}}_{v_{0}^{i}}\right|^{a} v_{0}^{i} \bar{W}^{k+1}\left(\tau_{i}, P_{v_{0}^{i}}, \mathbf{x}_{v_{0}^{i}}\right)$. We can distinguish several cases

1)if $\tau^{i}$ is a trivial tree, $a_{v_{0}^{i}}=0$ and $\left|\hat{\mathbf{x}}_{v_{0}^{i}}\right|^{a} v_{0}^{i} \bar{W}^{k+1}=\vec{v}_{k+1}$;

2)if $\tau^{i}$ is not a trivial tree:

2a) $a_{v_{0}^{i}}=0$. In this case we repeat word by word the analysis for $W^{k}$ leading from (3.15) to (3.19), with the trivial substitution $k \rightarrow k+1, v_{0} \rightarrow v_{0}^{i}$. Note that if $\mathcal{R} \neq 0$ the effect of the renormalization is of replacing in the truncated expectation in $(3.19) \tilde{\psi}^{(k+1)}\left(P_{v_{0}^{i}} \backslash Q_{v_{0}^{i}}\right)$ with $\hat{\psi}^{(k+1)}\left(P_{v_{0}^{i}} \backslash Q_{v_{0}^{i}}\right)$, defined in an analogous way as $\hat{\psi}\left(P_{v_{0}}\right)(3.16)$, and to produce a factor $\left(\mathbf{x}_{i}-\mathbf{x}_{j}\right)^{a_{v_{0}^{i}}}, \mathbf{x}_{i}, \mathbf{y}_{j} \in \mathbf{x}^{P_{v_{0}^{i}}}$ which is written as in (3.20).

2b) $a_{v_{0}^{i}} \neq 0$. In this case from (3.3) we have $\mathcal{R}=1$ if $\left|P_{v_{0}^{i}}\right| \geq 4$ and if $\left|P_{v_{0}^{i}}\right|=2$ we use (3.4). Also in this case we can repeat word by word the analysis for $W^{k}$ leading from (3.15) to (3.19), with the trivial substitutions $k \rightarrow k+1, v_{0} \rightarrow v_{0}^{i}$. The crucial point is that the propagators belonging to $T_{v_{0}^{i}}$ are of the form $\left(\mathbf{x}_{l}-\mathbf{y}_{l}\right)^{a_{l}} g\left(\mathbf{x}_{l} ; \mathbf{y}_{l}\right)$ with $a_{l} \leq 2$ i.e. the power $a$ is not increasing. In fact, in the case $\left|P_{v_{0}^{i}}\right|=4$ it could be a priori a factor $\left(\mathbf{x}_{l}-\mathbf{y}_{l}\right)$ for the action of $\mathcal{R}$ and another factor $\left(\mathbf{x}_{l}-\mathbf{y}_{l}\right)$ from $\left|\hat{\mathbf{x}}_{v_{0}^{i}}\right|_{v_{0}^{i}}^{a_{0}}$ but $\mathcal{R}=1$ from (3.3) and so the first factor is not present. Similar considerations for the $\left|P_{v_{0}^{i}}\right|=2$ case.

Iterating this procedure we find at the end

$$
\begin{gathered}
\mathcal{R} \hat{V}^{k}\left(\tau, \sqrt{Z_{k-1}} \psi^{(\leq k)}\right)=\int d \mathbf{x}_{v^{0}} \sum_{P_{v^{0}}} \sqrt{Z_{k-1}}\left|P_{v_{0}}\right| \tilde{\psi}^{(\leq k)}\left(P_{v_{0}}\right) \sum_{\left\{P_{v}\right\}}\left\{\int d t\right\} \\
{\left[\left.\prod_{\text {v not e.p. }}\left|\frac{Z_{h_{v}-1}}{Z_{h_{v}-2}}\right|\right|^{\left|P_{v}\right| / 2}\right]\left[\sum_{\{q\}}^{*} \prod_{\text {vot e.p. }} \frac{1}{s_{v} !} \tilde{\mathcal{E}}_{h_{v}}^{T}\left(\hat{\psi}^{\left(h_{v}\right)}\left(P_{v^{1}} \backslash Q_{v^{1}}\right), \ldots, \hat{\psi}^{\left(h_{v}\right)}\left(P_{v^{s}} \backslash Q_{v^{s}}\right)\right]\right.} \\
{\left[\prod_{i \in V_{1}}\left(\vec{v}_{h_{i}}\right)_{a} e^{2 i p_{F} x_{i} \gamma_{a}} \gamma^{h_{i} \delta_{a}}\right]\left[\prod_{i \in V_{2}} \hat{\varphi}_{m_{i}} e^{i 2 m_{i} p_{L} \mathbf{x}_{i}}\right]}
\end{gathered}
$$


where:

1)The symbol $\sum_{\left\{P_{v}\right\}}$ denotes the sum over all the compatible choices of the sets $P_{v}$ on all the vertex of $\tau$ except $v_{0}$; moreover $Q_{v} \subset P_{v}, P_{v}=\bigcup_{i} Q_{v^{i}}$.

2) $\hat{\psi}^{\left(h_{v}\right)}\left(P_{v} \backslash Q_{v}\right)=\prod_{f \in P_{v} \backslash Q_{v}} \partial^{q_{f}} \psi_{\mathbf{x}_{i j}(t)}^{\sigma(f)\left(h_{v}\right)}$ where $q_{f}=0,1,2,3$ with the same conventions as in (3.17). Moreover $\mathbf{x}_{i j}(t)=\sum_{i^{\prime}} \varepsilon_{i^{\prime} j}(t) \mathbf{x}_{i^{\prime} j}$ with $\sum_{i^{\prime}} \varepsilon_{i^{\prime} j(t)}=1$ and $\varepsilon_{i^{\prime} j}(0)=\delta_{i, i^{\prime}}$. Finally

$$
\tilde{\mathcal{E}}_{h}^{T}\left(\hat{\psi}\left(P_{1}\right), \ldots, \hat{\psi}\left(P_{k}\right)\right)=\sum_{T_{v}} \sum_{\{a\}}^{*}\left[\prod_{l \in T_{v}} \partial_{\mathbf{x}_{l}}^{q_{l}} \partial_{\mathbf{y}_{l}}^{q_{l}^{\prime}}\left[\left(\mathbf{x}_{l}-\mathbf{y}_{l}\right)^{a_{l}} g^{h}\left(\mathbf{x}_{l} ; \mathbf{y}_{l}\right)\right]\right] \int d P_{T}(s) \operatorname{det} G^{T}(s)
$$

where $\sum_{\{q\}}^{*}$ and $\sum_{\{a\}}^{*}$ have the following constraints. If $v$ is such that $\left|P_{v}\right|=4$ and the Kronecker $\delta$ of (2.26) is verified then $\hat{\psi}\left(P_{v}\right)$ contains a field $\partial \psi_{x_{i j}^{\prime}}$ and there is a line $\bar{l} \in T_{\bar{v}}$, $\bar{v}>v$, with $a_{\bar{l}}=1$ and for any $l \in T_{\hat{v}}, l \neq \bar{l}, \bar{v} \geq \hat{v}>v a_{l}=0$. If $v$ is such that $\left|P_{v}\right|=2$ and the Kronecker $\delta$ of (2.27) is verified then $\hat{\psi}\left(P_{v}\right)$ contains a field $\partial^{2} \psi_{\mathbf{x}_{i j}}$ or $\Delta \psi$ and there are two (possibly coinciding) lines $l_{1} \in T_{\bar{v}_{1}}, l_{2} \in T_{\bar{v}_{2}}$ with $\bar{v}_{1}>v, \bar{v}_{2}>v$ with $a_{l}=1$ and for any $l \in T_{\hat{v}}, l \neq l_{1}, l_{2} \bar{v}_{1} \geq \hat{v}>v, \bar{v}_{2} \geq \hat{v}>v$ one has $a_{l}=0$.

4) $\left\{\int d t\right\}$ is a product of integral over the interpolation parameters.

5) $V_{1}$ is the set of the end points of the tree, and $V_{2}$ is the set of end points not associated to running coupling constants. Moreover $\gamma_{a}= \pm 1$ in correspondence of end-points of kind $\tau$ or $\iota$ and zero otherwise, and $\delta_{a}=1$ in correspondence of end-points of kind $\nu$ and zero otherwise.

We sum (3.22) over the index $m_{i}$ i.e. we sum over all the trees differing only for the choices of the addend $\mathcal{R} V^{0}$ associated to a given end-point(i.e. we sum over trees with the same labels and differing only for the choices of $m_{i}$ ). We can bound $\operatorname{det} G^{h, T}$ by the well known Gramm-Hadamard inequality (see for instance [BGPS]). Once that we have bounded the determinant, we have to make the integration over the coordinates and over the interpolation variables. It is convenient to change variables from $\{\mathbf{x}\}$ to $\{\mathbf{r}\}$, where $\{\mathbf{r}\}$ is the collection of the difference $\mathbf{x}_{l}-\mathbf{y}_{l}$ appearing in the factors $\prod_{l} \partial_{\mathbf{x}_{l}}^{q_{l}} \partial_{\mathbf{y}_{l}}^{q_{l}^{\prime}}\left[\left(\mathbf{x}_{l}-\mathbf{y}_{l}\right)^{a_{l}} g^{h}\left(\mathbf{x}_{l} ; \mathbf{y}_{l}\right)\right]$. Note that $\mathbf{x}_{l} \equiv x_{i j}(t)$ so that the determinant of the Jacobian of this transformation is a function of $t$, and one can worry about its integrability. However it is possible to show (see [BM1], App. 3 ) that such determinant is exactly 1 . So we obtain a bound for $W^{k}(3.13)$, estimating the propagators by lemma 2.8 (taking $L \geq \gamma^{-h^{*}}$ we get $\left.\max \left\{\gamma^{h}, L^{-1}\right\}\right)=\gamma^{h}$ ), using lemma 2.6 and repeating analogous considerations for $\mathcal{L} \hat{V}^{k}\left(\tau, \sqrt{Z_{k-1}} \psi(\leq k)\right)$ :

$$
\begin{aligned}
& L \beta \gamma^{-k D\left(P_{v_{0}}\right)} \sum_{\left\{m_{i}\right\}} \sum_{\left\{P_{v}\right\}}\left[\prod_{\text {v not e.p. }} \mathbf{1}\left(\left|N_{v}\right| \geq \mathcal{A}\left(\gamma^{\frac{-h_{v}}{\tau}}\left|P_{v}\right|^{-\frac{1}{\tau}}-\bar{m}\left|P_{v}\right|\right)\right)\right]\left[\prod_{\text {v not e.p. }}\left|\frac{Z_{h_{v}-1}}{Z_{h_{v}-2}}\right|^{\frac{\left|P_{v}\right|}{2}}\right] \\
& C^{\left|Q_{v}\right|-\left|P_{v}\right|} J\left(\tau, P_{v_{0}}\right)\left[\prod_{\text {v not e.p. }} \gamma^{-\left[D\left(P_{v}\right)+z_{v}\left(N_{v}, P_{v}\right)\right]\left(h_{v}-h_{v^{\prime}}\right)}\right]\left[\prod_{i \in V_{1}}\left(\vec{v}_{h_{i}}\right)_{a}\right]\left[\prod_{i \in V_{2}}\left|\hat{\varphi}_{m_{i}}\right|\right]
\end{aligned}
$$

where the factor $\mathbf{1}\left(\left|N_{v}\right| \geq \mathcal{A}\left(\gamma^{\frac{-h_{v}}{\tau}}\left|P_{v}\right|^{-\frac{1}{\tau}}-\bar{m}\left|P_{v}\right|\right)\right)$ comes from lemma 2.7, as we know that the summand in (3.22) is vanishing if the condition in the indicator function is not verified, $C$ is a constant and

$$
J\left(\tau, P_{v_{0}}\right)=\mid \prod_{\text {v not e.p. }} \frac{1}{s_{v} !} \sum_{T_{v}} \int d t \prod_{l \in T} \int d\left\{\mathbf{r}_{l} \gamma^{2 h_{v}}\right\} \partial_{\mathbf{r}_{l}}^{q_{l}+q_{l}^{\prime}}\left[(\mathbf{r})_{l}^{a} \gamma^{-h_{v}}\left[\gamma^{-h_{v}} \bar{g}^{h_{v}}\left(\mathbf{r}_{l}\right)\right] \mid\right.
$$


and $D\left(P_{v}\right)=-2+\sum_{f \in P_{v}}\left(1 / 2+m_{f}\right)$, with $m_{f}$ being the order of derivatives applied to the field of label $f, v^{\prime}$ is the vertex preceding $v$ on the tree (so that $h_{v^{\prime}}=h_{v}-1$ ). The presence $z_{v}\left(N_{v}, P_{v}\right)$ is due to the renormalization procedure and it is defined as

$$
\begin{aligned}
& \text { 1) } z_{v}\left(N_{v}, P_{v}\right)=1 \text { if }\left|P_{v}\right|=4, \sum_{f \in P_{v}} m_{f}=0 \text { and } \sum_{f \in P_{v}} \sigma(f) \omega(f) p_{F}+2 N_{v} p_{L}=0 \\
& \text { 2) } z_{v}\left(N_{v}, P_{v}\right)=1 \text { if }\left|P_{v}\right|=2, \sum_{f \in P_{v}} m_{f}=1 \text { and } \sum_{f \in P_{v}} \sigma(f) \omega(f) p_{F}+2 N_{v} p_{L}=0 \\
& 3) z_{v}\left(N_{v}, P_{v}\right)=2 \text { if }\left|P_{v}\right|=2, \sum_{f \in P_{v}} m_{f}=0 \text { and } \sum_{f \in P_{v}} \sigma(f) \omega(f) p_{F}+2 N_{v} p_{L}=0
\end{aligned}
$$

Moreover $\int d\left\{\mathbf{r}_{l}\right\}=\sum_{x_{v} \in \Lambda} \int_{-\beta}^{\beta} \gamma^{\beta} d r_{0, l}$

It is easy to check that $J\left(\tau, P_{v_{0}}\right)$ is bounded, if $\beta, L \geq \gamma^{-h^{*}}$, by $\prod_{\mathrm{v} \text { not e.p. }} C^{\sum_{i}\left|P_{v^{i}}-\right| Q_{v^{i}} \mid}$

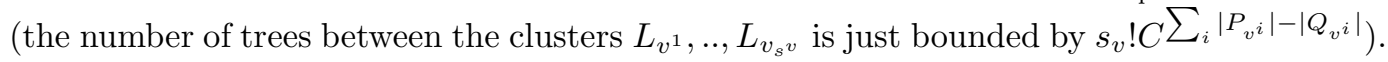

3.6 We can write then

$$
\begin{gathered}
\prod_{\text {v not e.p. }} \gamma^{-\left[D\left(P_{v}\right)+z_{v}\left(N_{v}, P_{v}\right)\right]\left(h_{v}-h_{v^{\prime}}\right)} \leq \\
\prod_{\text {v not e.p. }} \gamma^{\frac{-\left|P_{v}\right|}{6}}\left[\prod_{v \in T_{4}} \gamma^{h_{v}-h_{v^{\prime}}}\right]\left[\prod_{v \in T_{2}} \gamma^{h_{v}-h_{v^{\prime}}}\right]\left[\prod_{v \in T_{3}} \gamma^{2\left(h_{v}-h_{v^{\prime}}\right)}\right]
\end{gathered}
$$

where

1) $T_{4}$ is the set of vertices $v$ with $\left|P_{v}\right|=4, \sum_{f \in P_{v}} m_{f}=0$ and $\sum_{f \in P_{v}} \sigma(f) \omega(f) p_{F}+$ $2 N_{v} p_{L} \neq 0$

2) $T_{2}$ is is the set of vertices $v$ with $\left|P_{v}\right|=2, \sum_{f \in P_{v}} m_{f}=1$ and $\sum_{f \in P_{v}} \sigma(f) \omega(f) p_{F}+$ $2 N_{v} p_{L} \neq 0$

3) $T_{3}$ is is the set of vertices $v$ with $\left|P_{v}\right|=2, \sum_{f \in P_{v}} m_{f}=0$ and $\sum_{f \in P_{v}} \sigma(f) \omega(f) p_{F}+$ $2 N_{v} p_{L} \neq 0$.

Let be $\mathcal{T}$ the set of vertices $v$ (excluding end-points) such that $v^{\prime}$ is a non trivial vertex; we say that $v$ is a hard vertex. To an hard vertex $v$ we associate a depth defined in the following way: if $v$ is the first hard vertex (i.e. if $\bar{v}$ is any vertex on the tree following $v$, $\bar{v} \notin \mathcal{T})$ then $D_{v}=1$, otherwise $D_{v}=1+\max _{v^{\prime \prime}}\left\{D_{v^{\prime \prime}}\right\}$, where $v^{\prime \prime}$ are hard vertices following $v$ on the tree. Note that $D_{v} \leq-h_{v^{\prime}}+2$. We call $B_{D}$ the set of $v \in \mathcal{T}$ with depth $D$.

We can write

$$
\prod_{\text {v not e.p. }} \gamma^{-\left[D\left(P_{v}\right)+z_{v}\left(N_{v}, P_{v}\right)\right]\left(h_{v}-h_{v^{\prime}}\right)} \leq \prod_{\text {v not e.p. }} \gamma^{\frac{-\left|P_{v}\right|}{6}}\left[\prod_{v \in \mathcal{T}_{4}} \gamma^{-h_{v^{\prime}}}\right]\left[\prod_{v \in \mathcal{T}_{2}} \gamma^{-h_{v^{\prime}}}\right]\left[\prod_{v \in \mathcal{T}_{3}} \gamma^{\left.-2 h_{v^{\prime}}\right)}\right]
$$

where $v^{\prime}$ is the vertex preceding $v$ and $\mathcal{T}_{i}$ is the intersection between $\mathcal{T}$ and $T_{i}$. It is easy to show that

$$
\prod_{i \in V_{2}}\left|\hat{\varphi}_{m_{i}}\right| \leq e^{\frac{\xi n \bar{m}}{2}} \prod_{i \in V_{2}} e^{-\xi\left|m_{i}\right| / 2} \prod_{v \in \mathcal{T}} e^{\frac{-\xi\left|N_{v}\right|}{2 D_{v}+1}}
$$

In fact we write $\prod_{i \in V_{2}}\left|\hat{\varphi}_{m_{i}}\right| \leq\left[\prod_{i \in V_{2}} e^{-\xi\left|m_{i}\right| / 2}\right]\left[\prod_{i \in V_{2}} e^{-\xi\left|m_{i}\right| / 2}\right]$ and we want to show that

$$
\prod_{i \in V_{2}} e^{-\xi\left|m_{i}\right| / 2} \leq e^{\frac{\xi \bar{m} n}{2}}\left[\prod_{a=1}^{l} \prod_{v \in B_{a}} e^{\frac{-\xi\left|N_{v}\right|}{2^{D}+1}}\right]
$$


where $l \leq-k+2$. The 1.h.s. of (3.24) can be written as

$$
\begin{gathered}
{\left[\prod_{\substack{i \in V_{2} \\
i \notin L v, v \in B_{1}}} e^{-\xi\left|m_{i}\right| / 2}\right]\left[\prod_{v_{1} \in B_{1}} \prod_{i \in L_{v_{1}}} e^{\frac{-\xi\left|m_{i}\right|}{2}}\right] \leq} \\
e^{\frac{\xi \bar{m} n_{1}}{2}}\left[\prod_{\substack{i \in V_{2} \\
i \notin L, v \in B_{1}}} e^{-\xi\left|m_{i}\right| / 2}\right]\left[\prod_{v_{1} \in B_{1}} e^{\frac{-\xi\left|N_{v_{1}}\right|}{4}}\right]\left[\prod_{v_{1} \in B_{1}} e^{\frac{-\xi\left|N_{v_{1}}\right|}{4}}\right]
\end{gathered}
$$

where $i \in L_{v_{1}}$ are the end-points in the cluster $L_{v_{1}}$ and we used (3.10); $n_{1}$ are the end-point in $\cup_{v_{1} \in B_{1}} L_{v_{1}} v_{1} \in B_{1}$ of kinds $\iota, \tau$. The r.h.s of (3.25) can be bounded by

$$
\begin{gathered}
e^{\frac{\xi \bar{m} n_{1}}{2}}\left[\prod_{\substack{i \in V_{2} \\
i \notin L v}} e^{-\xi\left|m_{i}\right| / 2}\right]\left[\prod_{\substack{v_{1} \in B_{2} \\
L_{v_{1}} \not L_{v_{2}}, v_{2} \in B_{2}}} e^{\left.-\xi \frac{\left|N v_{1}\right|}{4}\right]}\right] \\
{\left[\prod_{v_{1} \in B_{1}} e^{\frac{-\xi\left|N_{v_{1}}\right|}{4}}\right]\left\{\prod _ { v _ { 2 } \in B _ { 2 } } [ \prod _ { v _ { 1 } : L _ { v _ { 1 } } \subset L _ { v _ { 2 } } } e ^ { \frac { - \xi | N _ { v _ { 1 } } | } { 4 } } ] \left[\prod_{i \in L_{v_{2}}}^{*} e^{\left.\left.\frac{-\xi\left|m_{i}\right|}{2}\right]\right\} \leq}\right.\right.} \\
e^{\frac{\xi \bar{m}\left(n_{1}+n_{2}\right)}{2}}\left[\prod_{\substack{\in \in V_{2} \\
i \notin L v}} e^{-\xi\left|m_{i}\right| / 2}\right]\left[\prod_{\substack{v_{1} \in B_{1} \cup B_{2} \\
L_{v_{1}} \not L_{v_{2}}, v_{2} \in B_{2}}} e^{-\frac{\xi\left|N_{v_{1}}\right|}{4}}\right] \\
{\left[\prod_{v_{1} \in B_{1}} e^{\left.\frac{-\xi\left|N_{v_{1}}\right|}{4}\right]}\right]\left[\prod_{v_{2} \in B_{2}} e^{\frac{-\xi\left|N_{v_{2} \mid}\right|}{8}}\right]\left[\prod_{v_{2} \in B_{2}} e^{\frac{-\xi\left|N_{v_{2}}\right|}{8}}\right]}
\end{gathered}
$$

where $n_{2}$ are the end-points in the clusters $\cup_{v_{2} \in B_{2}} L_{v_{2}}$ but not in any smaller ones contained in $v_{2}$ of kinds $\iota, \tau$, and $\prod_{i \in L_{v}}^{*}$ is the product over the end-points in $L_{v}$ not contained in any smaller cluster contained in $L_{v}$. Proceding in this way we have (3.24).

Using that $D_{v} \leq-h_{v^{\prime}}+2$ we have that, if $\mathcal{C}=\mathcal{T}_{2} \bigcup \mathcal{T}_{3} \bigcup \mathcal{T}_{4} \subset B$ and using (3.24)

$$
\prod_{\text {v not e.p. }} \mathbf{1}\left(\left|N_{v}\right| \geq \mathcal{A}\left(\gamma^{\frac{-h_{v}}{\tau}}\left|P_{v}\right|^{-\frac{1}{\tau}}-\bar{m}\left|P_{v}\right|\right)\right) \prod_{i \in V_{2}}\left|\hat{\varphi}_{m_{i}}\right| \leq C^{n} \prod_{i \in V_{2}} e^{-\xi\left|m_{i}\right| / 2} \prod_{v \in \mathcal{C}} e^{\frac{-\xi C_{2} \gamma}{2^{-h} \frac{-h_{v^{\prime}}}{\tau}}}
$$

where the constraint in the indicator function is used only for the $v \in \mathcal{C}$, and $\left|P_{v}\right| \leq 4$ for any $v \in \mathcal{C}$.

Then

$$
\begin{gathered}
\sum_{\left\{m_{i}\right\}}\left|\hat{V}^{k}\left(\hat{\tau}, \sqrt{Z_{k-1}} \psi^{\leq k}\right)\right| \leq C_{1}^{n} \varepsilon^{n} \gamma^{-k D\left(P_{v_{0}}\right)} \sum_{\left\{P_{v}\right\}} \prod_{\text {v not e.p. }} \gamma^{\frac{-\left|P_{v}\right|}{8}} \\
\prod_{v \in \mathcal{T}_{1}} \gamma^{-h_{v^{\prime}}} e^{\frac{-\xi C_{2} \gamma}{2^{-h_{v^{\prime}}+3}}} \prod_{v \in \mathcal{T}_{2}} \gamma^{-h_{v^{\prime}}} e^{\frac{-\xi C_{2} \gamma}{2^{-h} v_{v^{\prime}}+3}} \prod_{v \in \mathcal{T}_{3}} \gamma^{-2 h_{v^{\prime}}} e^{\frac{-\xi C_{2} \gamma}{2^{-h} v_{v^{\prime}}+3}}
\end{gathered}
$$

Choosing $\gamma$ so that $\gamma^{\frac{1}{\tau}} / 2>1$ and remembering that the hard vertices are $\leq C_{1} n$ we have that, for $i=1,2,3\left(C_{1}, C_{2}, C_{3}\right.$ are constants)

$$
\prod_{v \in \mathcal{T}_{i}} \gamma^{-2 h_{v^{\prime}}} e^{\frac{-\xi C_{2} \gamma}{2^{-h} \frac{-h_{v^{\prime}}}{\tau}}} \leq C_{2}^{n}
$$

By a standard calculation

$$
\sum_{\tau \in \tau_{k, n}} \sum_{\left\{P_{v}\right\} \text { v not e.p. }} \prod^{\frac{-\left|P_{v}\right|}{8}} \leq C_{3}^{n}
$$

and this completes the proof of Theorem 3.3. 
Remark: Formula (3.26) is the analogue of Brjuno lemma for this problem; it ensures that the small denominator problem arising in the series for the incommensurability of $\phi_{x}$, can be controlled taking into account the Diophantine condition. The same role is plaied by the original Brjuno lemma in the proof of the convergence of the Lindstedt series. The idea is quite simple: we can associate to each hard cluster with four or two external lines an exponential factor $e^{\frac{-\xi\left|N_{v}\right|}{2^{-h_{v^{\prime}}+3}}}$ which is indeed quite small if $\left|h_{v}\right|$ is big. It is due to the analyticity of the incommensurate potential $\varphi_{x}$. But $\left|N_{v}\right|$ has to be very large for the diophantine condition, as noted in lemma 2.7, and the resulting factor compensates the "bad" factors $\gamma^{-h_{v^{\prime}}}$ or $\gamma^{-2 h_{v^{\prime}}}$ due to the power counting. Note that the clusters which are not hard are clusters in which the external lines are not contracted, or are contracted with lines from the same cluster, so that it is sufficient to get the factor $e^{\frac{-\xi\left|N_{v}\right|}{2^{-h_{v^{\prime}}+3}}}$ for the hard clusters. The relevant or marginal terms in $(2.26),(2.27)$ are the analogue of the resonances in KAM theory.

We have now to perform the last integration (2.52).

3.7THEOREM If there exists constants $\kappa, C_{2}$, $\varepsilon$ such that $\frac{\left|\sigma_{h^{*}-1}\right|}{\gamma^{h^{*}}} \geq \kappa$ and $\left|\vec{v}_{h^{*}}\right| \leq \bar{\varepsilon}_{h^{*}}$, $\left|\frac{Z_{h^{*}}}{Z_{h^{*}-1}}\right| \leq e^{C_{2} \varepsilon_{h^{*}}^{2}}$ than

$$
\left|\int \tilde{P}_{Z_{h^{*}-1}}\left(d \psi^{\left(\leq h^{*}\right)}\right) e^{-\tilde{\mathcal{V}}^{h^{*}}\left(\sqrt{Z_{h^{*}}}\left(\leq h^{*}\right)\right.}\right| \leq C_{3} L \beta \gamma^{2 h^{*}}
$$

where $C_{3}$ is a suitable constant

PROOF The proof is rather straigthforward. In fact we can write (3.27) as a sum over trees, having only one non trivial vertex $v, h_{v} \equiv h^{*}$, and $s_{v}$ end points. Then (3.27) can be written as $\sum_{n=1}^{\infty} \sum_{\tau \in \tau_{n}} V^{\left(h^{*}\right)}(\tau)$, if $\tau_{n}$ are the trees with $n=s_{v}$ end points and $V^{h^{*}}(\tau)$ is given by

$$
\frac{1}{s_{v} !} E_{\leq h^{*}}^{T}\left[\int d \mathbf{x}_{v^{1}} \tilde{\psi}^{\left(\leq h^{*}\right)}\left(P_{v^{1}}\right) W^{h^{*}}\left(P_{v^{1}}, \mathbf{x}_{v^{1}}\right) \ldots \int d \mathbf{x}_{v^{s_{v}}} \tilde{\psi}^{\leq h^{*}}\left(P_{v^{s_{v}}}\right) W^{h^{*}}\left(P_{v^{s_{v}}}, \mathbf{x}_{v^{s_{v}}}\right)\right]
$$

where $E_{\leq h^{*}}^{T}$ denotes the integration with respect to the propagator $(2.53)$ and $W^{h^{*}}\left(\mathbf{x}_{v^{i}}\right)=$ $\sum_{\tau} W^{h^{*}}\left(\tau, P_{v^{i}}, \mathbf{x}_{v^{i}}\right)$ was estimated in Theorem 3.4. Using again the Gramm-Hadamard inequality we find

$$
\left|V^{h^{*}-1}(\tau)\right| \leq L \beta \varepsilon_{h^{*}}^{n} C^{n} \gamma^{2 h^{*}} \sum_{P_{v^{1}}, \ldots, P_{v^{s}}} \prod_{i=1}^{s_{v}}\left[\gamma^{h^{*}\left(\frac{\left|P_{v^{i}}\right|}{2}-2\right)} \gamma^{h^{*} \delta_{v^{i}}}\right]
$$

where $\delta_{v^{i}}=1$ if $\left|P_{v^{i}}\right|=2$ and it is zero otherwise, see Theorem 3.4, and the theorem follows.

\section{The flow of the Renormalization group}

4.1 The convergence of the expansion for the effective potential is proved by theorems 3.4,3.7 under suitable assumptions on the running coupling constants i.e. that there exists a finite $h^{*}(2.48)$ such that, given a constant $\bar{\varepsilon}_{k}$, then $\max _{k \geq h^{*}}\left|\vec{v}_{k}\right| \leq \bar{\varepsilon}_{k}$ and $\max _{k \geq h^{*}}\left|\frac{Z_{k}}{Z_{k-1}}\right| \leq$ $e^{c_{1} \bar{\varepsilon}_{k}^{2}}$. In this section we prove that it is possible to choose $|\lambda|, u$ so small and a proper $\nu$ so that the above conditions are indeed verified.

If $\vec{a}_{h}=\lambda_{h}, \sigma_{h}, \delta_{h}, \tau_{h}, \iota_{h}$ then

$$
\begin{gathered}
\vec{a}_{h-1}=\vec{a}_{h}+\vec{\beta}^{h}\left(\vec{a}_{h}, \nu_{h} ; \ldots ; \vec{a}_{0}, \nu_{0}\right) \\
\nu_{h-1}=\gamma \nu_{h}+\beta_{\nu}^{h}\left(\vec{a}_{h}, \nu_{h} ; \ldots ; \vec{a}_{0}, \nu_{0}\right)
\end{gathered}
$$


The property $\gamma>1$ can be used to show that such relations are equivalent to

$$
\begin{gathered}
\vec{a}_{h-1}=\vec{a}_{h}+\vec{\beta}^{h}\left(\vec{a}_{h} ; \ldots ; \vec{a}_{0} ; \nu_{h}\right) \\
\nu_{h-1}=\gamma \nu_{h}+\beta_{\nu}^{h}\left(\vec{v}_{h} ; \ldots ; \vec{v}_{0} ; \nu_{h}\right)
\end{gathered}
$$

The proof is a trivial adaptation to this case of app.4 of [BGPS]. For the parity of the propagator, $\beta_{\nu}^{h}=\nu_{h} \lambda_{h}^{2} \beta_{\nu}^{1, h}+\gamma^{h} R_{\nu}^{h},\left|\beta_{\nu}^{1, h}\right| \leq C,\left|R_{\nu}^{h}\right| \leq C \bar{\varepsilon}_{h}^{2}$. Moreover also from [BGPS], sec.7, it follows that it is possible to choose $\nu \equiv \nu(\lambda, u)$ so that $\nu_{h}=O\left(\gamma^{h}\right)$, for any choice of the running coupling constants such that $\max _{k \geq h^{*}}\left|\vec{v}_{k}\right| \leq \bar{\varepsilon}_{k}$ and $\max _{k \geq h^{*}}\left|\frac{Z_{k}}{Z_{k-1}}\right| \leq e^{c_{1} \bar{\varepsilon}_{k}^{2}}$. This is a version of the unstable manifold theorem.

We can write, for $h \geq h^{*}$

$$
\begin{gathered}
\lambda_{h-1}=\lambda_{h}+G_{\lambda}^{1, h}+G_{\lambda}^{2, h}+\gamma^{h} R_{\lambda}^{h} \\
\sigma_{h-1}=\sigma_{h}+G_{\sigma}^{1, h} \\
\delta_{h-1}=\delta_{h}+G_{\delta}^{1, h}+G_{\delta}^{2, h}+\gamma^{h} R_{\delta}^{h} \\
\tau_{h-1}=\tau_{h}+G_{\tau}^{2, h}+\gamma^{h} R_{\tau}^{h} \\
\iota_{h-1}=\iota_{h}+G_{\iota}^{2, h}+\gamma^{h} R_{\iota}^{h} \\
\frac{Z_{h-1}}{Z_{h}}=1+G_{z}^{1, h}+G_{z}^{2, h}+\gamma^{h} R_{z}^{h}
\end{gathered}
$$

where:

1) $G_{\lambda}^{1, h} \equiv G_{\lambda}^{1, h}\left(\lambda_{h}, \delta_{h} ; \ldots ; \lambda_{0}, \delta_{0}\right), G_{\delta}^{1, h} \equiv G_{\delta}^{1, h}\left(\lambda_{h}, \delta_{h} ; \ldots ; \lambda_{0}, \delta_{0}\right)$ and

$G_{z}^{1, h} \equiv G_{z}^{1, h}\left(\lambda_{h}, \delta_{h} ; \ldots ; \lambda_{0}, \delta_{0}\right)$ are given by series of terms involving only the Luttinger model part of the propagator $g_{L, \omega}^{(k)}(\mathbf{x} ; \mathbf{y}), k \geq h$, see lemma 2.4 .

2) $G_{\lambda}^{2, h}, G_{\delta}^{2, h}, G_{z}^{2, h}, G_{\tau}^{2, h}, G_{\iota}^{2, h}$ depend on all the running coupling constants and are given by a series of terms involving at least a propagator $C_{2}^{(k)}(\mathbf{x} ; \mathbf{y})$ or $g_{\omega,-\omega}^{(k)}(\mathbf{x} ; \mathbf{y}), k \geq h$. By lemma 2.4 if $C_{\alpha}$ is a suitable constant and $\hat{\varepsilon}_{h}=\max _{k \geq h}\left|g_{k}\right|$ :

$$
\left|G_{\lambda}^{2, h}\right|,\left|G_{\delta}^{2, h}\right|,\left|G_{z}^{2, h}\right|,\left|G_{\tau}^{2, h}\right|,\left|G_{\iota}^{2, h}\right| \leq C_{\alpha} \hat{\varepsilon}_{h}^{2}\left|\frac{\sigma_{h}}{\gamma^{h}}\right|
$$

3) By a second order computation one obtains

$$
G_{\sigma}^{1, h}=\sigma_{h} g_{h}\left[-\beta_{1}+\bar{G}_{\sigma}^{1, h}\right] \quad G_{z}^{1, h}=g_{h}^{2}\left[\beta_{2}+\bar{G}_{z}^{1, h}\right]
$$

with $\beta_{1}, \beta_{2}$ non vanishing positive contants and $\left|\bar{G}_{\sigma}^{1, h}\right| \leq C_{\alpha} \hat{\varepsilon}_{h}$ and $\left|\bar{G}_{z}^{1, h}\right| \leq C_{\alpha} \hat{\varepsilon}_{h}$. Moreover $G_{\lambda}^{1, h}, G_{\delta}^{1, h}$ are vanishing at the second order.

4) $R_{i}^{h}, i=\lambda, z, \delta, \tau, \iota$ depend on all the running coupling constants and there is at least a propagator $C_{2}^{(k)}(\mathbf{x} ; \mathbf{y}), k \geq h .\left|R_{i}^{h}\right| \leq C_{\alpha} \bar{\varepsilon}_{h}^{2}$.

4.2LEMMA: There exist positive constants $c_{1}, c_{2}, c_{3}, c_{4}$ such that, if $\lambda, u$ are small enough and $h \geq h^{*}$ :

$$
\begin{gathered}
\left|\lambda_{h-1}-\lambda_{0}\right|<|\lambda|^{3 / 2} \\
\lambda \beta_{2} c_{3} h<\log \left(\frac{\left|\sigma_{h-1}\right|}{\left|\sigma_{0}\right|}\right)<\lambda \beta_{2} c_{4} h \\
-\beta_{1} c_{1} \lambda^{2} h<\log \left(\left|Z_{h-1}\right|\right)<-\beta_{1} c_{2} \lambda^{2} h \quad\left|\tau_{h-1}-\tau_{0}\right|<|\lambda|^{3 / 2}
\end{gathered}
$$




$$
\left|\iota_{h-1}-\iota_{0}\right|<|\lambda|^{3 / 2} \quad\left|\delta_{h-1}-\delta_{0}\right|<|\lambda|^{3 / 2}
$$

PROOF We proceed by induction. Assume that the above inequalities hold for any $k \geq h$ and we prove them for $h-1$. Then by (4.5)

$$
\left(1-\beta_{2} \lambda_{h}-c_{a} \lambda^{2}\right) \leq \frac{\sigma_{h-1}}{\sigma_{h}} \leq\left(1-\beta_{2} \lambda_{h}+c_{b} \lambda^{2}\right)
$$

with $c_{b}, c_{a}>0$ are suitable constants. Then, if $\lambda>0$ (for fixing ideas) there exists a $c_{4}<1$ such that

$$
\left|\sigma_{h-1}\right| \leq\left|\sigma_{0}\right| \gamma^{c_{4} \lambda \beta_{2}(h-1)} \frac{\left(1-\beta_{2} \lambda+c_{b} \lambda^{2}\right)}{\gamma^{-\lambda c_{4} \beta_{2}}} \leq\left|\sigma_{0}\right| \gamma^{\lambda c_{4} \beta_{2}(h-1)}
$$

and a $c_{3}>1$ such that

$$
\left|\sigma_{h-1}\right| \geq\left|\sigma_{0}\right| \gamma^{c_{3} \lambda \beta_{2}(h-1)} \frac{\left(1-\beta_{2} \lambda-c_{a} \lambda^{2}\right)}{\gamma^{-\lambda c_{3} \beta_{2}}} \geq\left|\sigma_{0}\right| \gamma^{c_{4} \lambda \beta_{2}(h-1)}
$$

We proceed in the same way for $Z_{h}$. Moreover let be $\mu_{h}=\left(g_{h}, \delta_{h}\right)$ and we write

$$
G_{\mu}^{1, h}\left(\mu_{h}, \mu_{h+1}, \ldots, \mu_{0}\right)=G_{\mu}^{1, h}\left(\mu_{h}, \ldots, \mu_{h}\right)+\sum_{k=h+1}^{0} D^{h, k}
$$

where

$$
D^{h, k}=G_{\mu}^{1, h}\left(\mu_{h} \ldots, \mu_{h}, \mu_{k}, \mu_{k+1}, . . \mu_{0}\right)-G_{\mu}^{1, h}\left(\mu_{h}, \ldots, \mu_{h}, \mu_{h}, \mu_{k+1}, \ldots, \mu_{0}\right)
$$

It holds that

$$
G_{\mu}^{1, h}\left(\mu_{h}, \ldots, \mu_{h}\right)=O\left(\gamma^{h}\right)
$$

This remarkable cancellation is due the fact that, by definition, $G_{\mu}^{1, h}\left(\mu_{h}, \ldots, \mu_{h}\right)$ is identical to the corresponding quantity of the Luttinger model for which (4.9) is proved (see eq.(7.6) of [BGPS], as a consequence of the analyticity of the Luttinger model Beta function and of some properties of the exact solution, see [BeGM],[BM1]). On the other hand, by the estimates of sec. 3

$$
\left|D^{h, k}\right| \leq C \lambda^{2} \gamma^{-\frac{k-h}{2}}
$$

so that

$$
\left|\mu_{h-1}-\mu_{h}\right| \leq C \lambda^{2} \sum_{k=h}^{0} \gamma^{-\frac{k-h}{2}}+C \lambda^{2} \sum_{k=h}^{0} \frac{\left|\sigma_{k}\right|}{\gamma^{k}}+\lambda^{2} C \sum_{k=h}^{0} \gamma^{k}
$$

and the lemma follows noting that, as $h \geq h^{*}$

$$
\sum_{k=h}^{0} \frac{\left|\sigma_{k}\right|}{\gamma^{k}} \leq\left|\sigma_{h^{*}}\right| \gamma^{-h^{*}} \sum_{k=h}^{0} \gamma^{-k+h^{*}} \frac{\left|\sigma_{k}\right|}{\sigma_{h^{*}}} \leq K
$$

if $K$ is a constant. For $\tau_{h}, \iota_{h}$ we proceed in a similar way

As a corollary of the above estimates on the running coupling constants we find that $h^{*}$ is finite:

$$
\frac{\log _{\gamma}\left|\sigma_{0}\right|}{1-\lambda \beta_{2} c_{\alpha}} \leq h^{*} \leq \frac{\log _{\gamma}\left|\sigma_{0}\right|+1}{1-\lambda \beta_{2} c_{\beta}}
$$

for some positive constants $c_{\alpha}, c_{\beta}$. From lemma 4.2 it follows that we can choose $\lambda$, $u$ so small so that the conditions for the validity of theorems 4.3,3.7 are indeed fulfilled; in particular 
one can choose $L, \beta \geq \gamma^{-h^{*}}$. This concludes the proof of the convergence of the expansion for the effective potential.

4.3 From the proof of the convergence of the effective potential and the relative bounds it is a standard matter to deduce the bounds for the expansion of the two point Schwinger function; we refer for this to [BGPS], sec.6. It holds that

$$
S(x, y)=S^{u \cdot v \cdot}(\mathbf{x} ; \mathbf{y})+\sum_{h=h^{*}}^{0} \sum_{\omega_{1}, \omega_{2}} e^{i p_{F}\left(\omega_{1} x-\omega_{2} y\right)} \cdot\left(\frac{g_{\omega_{1}, \omega_{2}}^{(h)}(\mathbf{x} ; \mathbf{y})}{Z_{h}}+\frac{\bar{S}_{\omega_{1}, \omega_{2}}^{(h)}(\mathbf{x} ; \mathbf{y})}{Z_{h}}\right)
$$

where we call $g^{\left(\leq h^{*}\right)}(\mathbf{x} ; \mathbf{y})$ simply $g^{\left(h^{*}\right)}(\mathbf{x} ; \mathbf{y})$ and the first addend is obtained by the integration of $\psi^{(1)}$ ( and it is bounded by $\frac{C_{N}}{1+|\mathbf{x}-\mathbf{y}|^{N}}$ ); $\bar{S}_{\omega_{1}, \omega_{2}}^{(h)}(\mathbf{x} ; \mathbf{y})$ is given by an expansion similar to the one in sec.3 and one can check that, for $L, \beta \geq \gamma^{-h^{*}},\left|\bar{S}_{\omega_{1}, \omega_{2}}^{(h)}\right| \leq A \bar{\varepsilon}_{h} \gamma^{h} \frac{C_{N}}{1+\left(\gamma^{h}|\mathbf{x}-\mathbf{y}|^{N} N\right.}$, $\bar{\varepsilon}_{h}=\sup _{k>h}\left|\vec{v}_{k}\right|$, see [BGPS]..

We are now ready to prove the main theorem in 4.1. First of all, we note that the r.h.s. of (4.11) has a meaning also if we take the formal limit $i \rightarrow \infty, \beta \rightarrow \infty$, (recall that $L=L_{i}$ ), by doing the substitution

$$
\frac{1}{L_{i} \beta} \sum_{\mathbf{k} \in \mathcal{D}_{L_{i}, \beta}} \rightarrow \int_{T^{1} \times R} \frac{d \mathbf{k}}{(2 \pi)^{2}} .
$$

The substitution (4.12), applied to (4.11), allows to define $g^{(h)}(\mathbf{x} ; \mathbf{y})$ in the limit $i \rightarrow \infty$, $\beta \rightarrow \infty$, at least for $h \leq 0$. For $h=1$, one has to be careful, since the integral over $k_{0}$ is not absolutely convergent. However it is easy to prove, by using standard well known arguments, that the limit as $i \rightarrow \infty$ and $\beta \rightarrow \infty$ of $g^{(1)}(\mathbf{x} ; \mathbf{y})$ is well defined for $x_{0} \neq y_{0}$ and has the same discontinuity in $x_{0}=y_{0}$ of the same limit taken on the free propagator (2.1). The previous considerations says that, for $\lambda, u$ real and small enough, there exists the limit

$$
S(\mathbf{x} ; \mathbf{y})=\lim _{\substack{\beta \rightarrow \infty \\ i \rightarrow \infty}} S^{L_{i}, \beta}(\mathbf{x} ; \mathbf{y})
$$

and that this limit is obtained by doing the substitution (4.12) in all quantities appearing in the r.h.s. of (4.11). 4.4 Let us define

$$
\eta_{1}=-\frac{\log \left(Z_{h^{*}}\right)}{\log \left|u \hat{\varphi}_{\bar{m}}\right|} \quad \eta_{2}=-1+\frac{\log \left(\sigma_{h^{*}}\right)}{\log \left|u \hat{\varphi}_{\bar{m}}\right|}
$$

which are respectively $\beta_{2} \lambda^{2}+O\left(\lambda^{3}\right)$ and $\beta_{1} \lambda+O\left(\lambda^{2}\right)$. We call $Z_{h^{*}}=\hat{Z}$ and $\sigma_{h^{*}}=\hat{u}$, see (1.6). The bounds (1.7),(1.8) can be obtained by (4.11). In fact if $1 \leq|\mathbf{x}-\mathbf{y}| \leq \hat{u}^{-1}$ it holds, for $\gamma^{-h_{x}-1}<|x-y| \leq \gamma^{-h_{x}}, h_{x}>h^{*}$,

$$
\left.\sum_{h=0}^{h^{*}} \mid \frac{g_{\omega_{1}, \omega_{2}}^{(h)}(\mathbf{x} ; \mathbf{y})}{Z_{h}}+\frac{\bar{S}_{\omega_{1}, \omega_{2}}^{(h)}(\mathbf{x} ; \mathbf{y})}{Z_{h}}\right) \mid \leq A_{1}\left[\sum_{h=h^{*}}^{h_{x}-1} \frac{\gamma^{h}}{Z_{h}}+\sum_{h=h_{x}}^{0} \frac{\gamma^{h}}{Z_{h}} \frac{C_{N}}{\gamma^{N h}|x-y|^{N}}\right] \leq A_{2} \gamma^{h_{x}\left(1-\eta_{3}\right)}
$$

On the other hand if $|\mathbf{x}-\mathbf{y}| \geq \hat{u}^{-1}$ :

$$
\begin{aligned}
\sum_{h=0}^{h^{*}} \mid \frac{g_{\omega_{1}, \omega_{2}}^{(h)}(\mathbf{x} ; \mathbf{y})}{Z_{h}} & \left.+\frac{\bar{S}_{\omega_{1}, \omega_{2}}^{(h)}(\mathbf{x} ; \mathbf{y})}{Z_{h}}\right) \mid \leq \frac{C_{N}}{|x-y|^{N}} \sum_{h=0}^{h^{*}} \frac{\gamma^{-(N-1) h}}{Z_{h}} \\
& \leq A_{2} \frac{\gamma^{h^{*}}}{Z_{h^{*}}} \frac{C_{N}}{\left(\gamma^{h^{*}}|x-y|\right)^{N}}
\end{aligned}
$$

if $A_{1}, A_{2}$ are positive constants. From the above bounds and lemma 2.6 it follows that

$$
\sum_{h=0}^{h^{*}} \sum_{\omega_{1}, \omega_{2}} e^{-i p_{F}\left(\omega_{1} \mathbf{x}-\omega_{2} \mathbf{y}\right)} \frac{g_{\omega_{1}, \omega_{2}}^{(h)}(\mathbf{x} ; \mathbf{y})}{Z_{h}}-\sum_{\omega} e^{i p_{F}(\omega(x-y))} \frac{1}{(L \beta)} \sum_{\mathbf{k}^{\prime} \in \mathcal{D}_{L, \beta}} \frac{f_{h}\left(\mathbf{k}^{\prime}\right)}{-i k_{0}+\omega k^{\prime}} \frac{1}{\left|\mathbf{k}^{\prime}\right|^{\eta_{3}}} \leq
$$




$$
C \sum_{h=0}^{h^{*}}\left[\frac{\sigma_{h}}{Z_{h}}\right] \leq C_{1} \frac{O(u, \hat{u})}{\hat{Z}} \log \hat{u}
$$

so proving (1.9).

4.5 Let us prove the decomposition (1.11). By diagonalizing the quadratic form $\frac{g^{(h)}(\mathbf{x}, \mathbf{y})}{Z_{h-1}}=$ $\sum_{\omega_{1}, \omega_{2}} \frac{e^{-i p_{F}\left(\omega_{1} x-\omega_{2} y\right)}}{Z_{h-1}} g_{\omega_{1}, \omega_{2}}^{(h)}(\mathbf{x} ; \mathbf{y})$ it is possible to see that, from (2.33):

$$
\frac{g^{(h)}(\mathbf{x} ; \mathbf{y})}{Z_{h-1}}=\frac{1}{(L \beta)} \sum_{\mathbf{k}^{\prime} \in \mathcal{D}_{L, \beta}} e^{-i \mathbf{k}^{\prime}(\mathbf{x}-\mathbf{y})} \frac{\tilde{f}_{h}\left(k^{\prime}\right)}{Z_{h-1}}\left[\frac{F_{x y}\left(k^{\prime}, \sigma_{h-1}\right)}{A+B}+\frac{F_{x y}\left(-k^{\prime},-\sigma_{h-1}\right)}{A-B}\right]
$$

where

$$
\begin{gathered}
F_{x y}\left(k^{\prime}, \sigma_{h}\right)=\hat{\phi}\left(k^{\prime}, x, \sigma_{h}\right) \hat{\phi}\left(k^{\prime},-y, \sigma_{h}\right) \\
\hat{\phi}\left(k^{\prime}, x, \sigma_{h}\right)=\frac{1}{\sqrt{2 B}}\left[\sqrt{B-C} e^{i p_{F} \mathbf{x}}-\frac{e^{-i p_{F} x}}{\sqrt{B-C}}\right]
\end{gathered}
$$

and $A=-i k_{0}+\cos \left(p_{F}\right)\left(1-\cos k^{\prime}\right), B=\sqrt{\left(C^{2}+\sigma_{h}^{2}\right)}$ and $C=v_{0} \sin k^{\prime}$. We can rewrite the above integral in terms of the $k$ variable. Recall that, if $\omega=\operatorname{sign}(k)$ and $f_{h}\left(\mathbf{k}^{\prime}\right) \neq 0$, then $k=\omega p_{F}+k^{\prime}$. Hence

$$
\begin{gathered}
\frac{g^{(h)}(\mathbf{x} ; \mathbf{y})}{Z_{h-1}}=\sum_{\omega_{1}, \omega_{2}} \frac{e^{i p_{F}\left(\omega_{1} x-\omega_{2} y\right)}}{Z_{h-1}} g_{\omega_{1}, \omega_{2}}^{h}(\mathbf{x} ; \mathbf{y})= \\
=\frac{1}{(L \beta)} \sum_{\mathbf{k} \in \mathcal{D}_{L, \beta}} \frac{\tilde{f}_{h}(\mathbf{k})}{Z_{h-1}} \frac{\left.\phi\left(k, x, \sigma_{h-1}\right)\right) \phi\left(k,-y, \sigma_{h-1}\right) e^{i k_{0}\left(x_{0}-y_{0}\right)}}{-i k_{0}-\left(\varepsilon\left(k, \sigma_{h-1}\right)-p_{F}^{2}\right)}
\end{gathered}
$$

where $\varepsilon\left(k, \sigma_{h}\right), \phi\left(k, x, \sigma_{h}\right)$ are defined in sec.(1.12). Inserting (4.14) in (4.11) one obtains the decomposition (1.11). For clarity of exposition we have preferred to write such formula avoiding the use of the scales. It is clear that the $h$-dependence of $\sigma_{h-1}, Z_{h-1}$ can be seen as a momentum dependence, for the compact support properties of $\tilde{f}_{h}(\mathbf{k})$. The functions $\hat{u}(\mathbf{k}), \hat{Z}(\mathbf{k})$ are defined in the following way: they are equal to $\sigma_{h-1}, Z_{h-1}$ for $\gamma^{h} \leq\left|\mathbf{k}^{\prime}\right|<$ $\gamma^{h+1}$ if $h \geq h^{*}$, and to $\sigma_{h^{*}}, Z_{h^{*}}$ for $\left|\mathbf{k}^{\prime}\right| \leq \gamma^{h^{*}}$. With this definition the difference between (1.12) and (4.14) is in the second addend of the decomposition (1.11) (remembering that, from (4.5), $\frac{\sigma_{h-1}}{\sigma_{h}}=1+O(\lambda)$ ).

4.6 Finally the lower bound on the spectral gap can be obtained showing that we can prove the boundedness on $S\left(x, y ; k_{0}\right)$ for $k_{0}$ complex, for $\left|\operatorname{Im}\left(k_{0}\right)\right| \leq \frac{\sigma_{h^{*}}}{2}$. The expansion disussed before is not suitable for this, as the functions $\hat{f}_{h}(\mathbf{k})$ are not analytic in $k_{0}$. However one can repeat the above analysis using a decomposition $\hat{f}_{h}(k)$ i.e. not depending on $k_{0}$ (see [BGM1] app.4 in which are discussed the (obvious) modifications in the bounds changing the cut-off functions). So in the support of $\hat{f}_{h}(k)$, for $h>h^{*}$ we have that, if $|\eta| \leq \frac{\sigma_{h^{*}}}{2}$

$$
\mid \operatorname{Re}\left[A_{h}\left(k^{\prime}, k_{0}+i \eta\right] \mid \geq a_{0} \gamma^{2 h}+\sigma_{h}^{2}-\eta^{2} \geq c_{1} \gamma^{2 h}\right.
$$

as, by (2.48), $\gamma^{h}>\gamma^{h^{*}} \geq \frac{4 \sigma_{h^{*}}}{a_{0} \gamma}$. On the other hand if $h \leq h^{*}$

$$
\mid \operatorname{Re}\left[A_{h}\left(k^{\prime}, k_{0}+i \eta\right] \mid \geq \frac{c_{2}}{\gamma^{h^{*}}}\right.
$$

for some constant $c_{1}, c_{2}$, so everything is essentially unchanged.

Acknowledgements I thank G.Benfatto and G.Gallavotti for many important discussions. 


\section{References}

[A] S. Aubry in Polarons and Bipolarons in Hich $T_{c}$ superconductors and related materials, editors Salye E, Alexandrov A., Liang W.

[AAR] S. Aubry, G. Abramovici, J. Raimbaut: Chaotic polaronic and bipolaronic states in the adiabatic Holstein model, J. Stat. Phys. 67, 675-780 (1992).

[B] A.D. Bryuno: The analytic form of differential equations I, Trans. Moscow Math. Soc. 25, 131-288 (1971); II, Trans. Moscow Math. Soc. 26, 199-239 (1972).

[BG] G.Benfatto, G.Gallavotti: Perturbation theory of the Fermi surface in a quantum liquid. A general quasiparticle formalism and one dimensional systems, J. Stat. Phys. 59, 541-664 (1990).

[BGL] G.Benfatto, G.Gallavotti, J. Lebowitz: Disorder in the 1D Holstein model, Hel.Phys. Acta (1992).

[BGM1] G.Benfatto,G.Gentile,V.Mastropietro: J. Stat. Phys.,89,655-708 (1997).

[BGM2] G.Benfatto,G.Gentile,V.Mastropietro: preprint (1997).

[BGPS] G.Benfatto, G.Gallavotti, A.Procacci, B.Scoppola: Beta functions and Schwinger functions for a many fermions system in one dmension, Comm.Math.Phys. 160, 93-171 (1994).

[BM1] F.Bonetto,V.Mastropietro: Beta function and anomaly of the Fermi surface for a $\mathrm{d}=1$ system of interacting fermions in a periodic potential, Comm.Math.Phys. 172, 57-93 (1995).

[BM2] F.Bonetto,V.Mastropietro: Filled band Fermi systems Mat.Phys.Elect.Jour 2, 1-43 (1996).

[BM3] F.Bonetto,V.Mastropietro: Critical indices in a d=1 filled band Fermi system, Phys. Rev. B 56,3,1296-1308 (1997).

[BM4] F.Bonetto,V.Mastropietro: Critical indices for the Yukawa2 quantum field theory, Nucl.Phys.B 497, 541-554 (1997).

[BeGM] G.Benfatto, G.Gallavotti,V.Mastropietro: Renormalization group and the Fermi surface in the Luttinger model, Phys. Rev. B 45, 5468-5480, (1992).

[BLT] J. Belissard, R. Lima, D. Testard: A metal-insulator transition for almost Mathieu model, Comm. Math. Phys. 88, 207-234 (1983).

[CF] L. Chierchia, C. Falcolini: A direct proof of a theorem by Kolmogorov in hamiltonian systems, Ann. Scuola Norm. Super. Pisa Cl. Sci. 21, No. 4, 541-593 (1995).

[D] H. Davenport: The Higher Arithmetic, Dover, New York, 1983.

[DS] E.I. Dinaburg, Ya.G. Sinai: On the one dimensional Schroedinger equation with a quasiperiodic potential, Funct. Anal. and its Appl. 9, 279-289 (1975).

[E] L.H. Eliasson: Floquet solutions for the one dimensional quasi periodic Schroedinger equation, Comm. Math. Phys. 146, 447-482 (1992).

[E1] L.H. Eliasson: Absolutely convergent series expansions for quasi-periodic motions, Report 2-88, 1-31, Department of Mathematics, University of Stockholm (1988) and Mat.Phys.Elect.Jour 2 .

[FMRS] J. Feldman, J.Magnen, V.Rivasseau, E.Trubowitz: An infinite volume expansion for many fermions Freen functions, Helv.Phys.Acta. 65 679-721,(1992)

[G1] G. Gallavotti: Twistless KAM tori, Comm. Math. Phys. 164, 145-156 (1994).

[G2] G. Gallavotti: Renormalization group and ultraviolet stability for scalar fields via renormalization group methods Rev. Mod. Phys. 57 471-562 (1985)

[GM] G. Gentile, V. Mastropietro: Methods for the analysis of the Lindstedt series for KAM tori and renormalizability in classical mechanics. A review with some applications, Rev. Math. Phys. 8, 393-444 (1996).

[H] T. Holstein: Studies of polaron motion, part 1. The molecular-crystal model. Ann. Phys. 8, 325-342, (1959).

[JM] R.A. Johnson, J. Moser: The rotation number for almost periodic potentials, Commun. Math. Phys. 84, 403-438 (1982).

[KL] T. Kennedy, E.H. Lieb: An itinerant electron model with crystalline or magnetic long range order, Physica A 138, 320-358 (1986).

[L] Lee P.A. Nature 291,11-12,1981. 
[ML] V.Mastropietro: Interacting soluble Fermi systems in one dimension, Nuovo Cim.B 1, 304-312, (1994).

[ML] D.Mattis, E.Lieb: Exact solution of a many fermion system and its associated boson field, Journ.Math.Phys. 6, 304-312, (1965).

[MP] J. Moser, J. Pöschel: An extension of a result by Dinaburg and Sinai on quasi periodic potentials, Comment. Math. Helv. 59, 39-85 (1984).

[NO] J.W. Negele, H. Orland: Quantum many-particle systems, Addison-Wesley, New York, 1988.

[PF] L. Pastur, A. Figotin: Spectra of random and almost periodic operators, Springer, Berlin, 1991.

[P] R.E. Peierls: Quantum theory of solids, Clarendon, Oxford, 1955. 